International Journal of Uncertainty,

Fuzziness and Knowledge-Based Systems

Vol. 15, No. 1 (2007) 13-41

(C) World Scientific Publishing Company

\title{
A BILATTICE-BASED FRAMEWORK FOR HANDLING GRADED TRUTH AND IMPRECISION
}

\author{
GLAD DESCHRIJVER \\ Department of Applied Mathematics and Computer Science, Ghent University, \\ Krijgslaan 281 (S9), B-9000 Gent, Belgium \\ Glad.Deschrijver@UGent.be \\ OFER ARIELI \\ Department of Computer Science, The Academic College of Tel-Aviv \\ Antokolski 4, Tel-Aviv 61161, Israel \\ oarieli@mta.ac.il \\ CHRIS CORNELIS \\ Department of Applied Mathematics and Computer Science, Ghent University, \\ Krijgslaan 281 (S9), B-9000 Gent, Belgium \\ *Chris.Cornelis@UGent.be \\ ETIENNE E. KERRE \\ Department of Applied Mathematics and Computer Science, Ghent University, \\ Krijgslaan 281 (S9), B-9000 Gent, Belgium \\ †Etienne.Kerre@UGent.be
}

Received 5 December 2005

Revised 24 November 2006

\begin{abstract}
We present a family of algebraic structures, called rectangular bilattices, which serve as a natural accommodation and powerful generalization to both intuitionistic fuzzy sets (IFSs) and interval-valued fuzzy sets (IVFSs). These structures are useful on one hand to clarify the exact nature of the relationship between the above two common extensions of fuzzy sets, and on the other hand provide an intuitively attractive framework for the representation of uncertain and potentially conflicting information. We also provide these structures with adequately defined graded versions of the basic logical connectives, and study their properties and relationships. Application potential and intuitive appeal of the proposed framework are illustrated in the context of preference modeling.
\end{abstract}

Keywords: Bilattices; intuitionistic fuzzy sets; interval-valued fuzzy sets; graded logical connectives; preference modeling.

\section{Introduction}

As is well-known, fuzzy logic is aimed at handling the concept of partial truth between 'completely true' and 'completely false' by drawing truth degrees from the 
unit interval $[0,1]$ (or, more generally, from a complete lattice $\mathcal{L}$, as in Goguen's $\mathcal{L}$-fuzzy $\operatorname{logic}^{1}$ ). Its importance derives from the fact that most modes of human reasoning and especially commonsense reasoning are approximate in nature rather than exact.

Fuzziness, however, cannot adequately cover all the imperfections inherent to real-life situations, since the 'one-dimensional' ordering of truth degrees cannot cope with information deficiency (i.e., a lack or excess of information). In previous papers, ${ }^{2-4}$ we have already argued that bilattices (due to Ginsberg ${ }^{5}$ and then Fitting ${ }^{6-9}$ ) can be used to complement the common notion of graded truth (or membership) from $(\mathcal{L}$-)fuzzy set theory, with a notion of graded knowledge. In this sense, bilattices offer a naturally attractive candidate framework for representing graded, uncertain and potentially conflicting information, a claim that is supported also in Refs. 10, 11, 12 .

In this paper, we vindicate this thesis by showing that so-called rectangular bilattices provide an elegant framework for bridging between intuitionistic fuzzy sets (IFSs) ${ }^{13}$ and interval-valued fuzzy sets (IVFSs), ${ }^{14}$ which are two frequently encountered and syntactically equivalent generalizations of Zadeh's fuzzy sets. In particular, we will show that Atanassov's decision to restrict the evaluation set for $\mathcal{L}$-intuitionistic fuzzy sets to consistent couples of the "square" $\mathcal{L}^{2}$ forces the resulting structure to coincide with the "triangle" $\mathcal{I}(\mathcal{L})$ (see Section 2.3).

In the sequel, the developed framework is equipped with suitable implementations for the common logical connectives of negation, conjunction, disjunction and implication. We also demonstrate the robustness of our framework in the context of preference modeling, and show that (rectangular) bilattices are useful not only for encoding the problem statement, but also for its generic solution strategy.

The remainder of this paper is organized as follows: in Section 2 we recall some basic definitions regarding the underlying structures of our framework, namely: bilattices, rectangular bilattices, and derived from them squares and triangles. Then, in Section 3, we show the relationships of these structures to intuitionistic fuzzy sets and interval-valued fuzzy sets. In Section 4 we consider graded versions of the basic logical connectives for reasoning with uncertainty in our setting, and in Section 5 we discuss the application potential of our framework in the context of modeling imprecise preference information. Finally, in Section 6 we conclude.

\section{Preliminaries}

\subsection{Bilattices}

Definition 1. A bilattice ${ }^{5}$ is a triple $\mathcal{B}=\left(B, \leq_{t}, \leq_{k}\right)$, where $B$ is a nonempty set containing at least two elements, and $\left(B, \leq_{t}\right),\left(B, \leq_{k}\right)$ are complete lattices. ${ }^{1}$

\footnotetext{
${ }^{1}$ Structures that meet this definition are sometimes called pre-bilattices. In such cases the notion 'bilattices' is reserved for some particular type of pre-bilattices which is determined according to the way the two partial orders are related; see Definition 2.
} 
The two partial orders $\leq_{t}$ and $\leq_{k}$ of bilattices intuitively represent differences in the degree of truth and in the amount of knowledge/information (respectively), conveyed by the assertions. In the sequel, following the usual notations for the basic bilattice operations, we shall denote by $\wedge$ (respectively, by $\vee$ ) the $\leq_{t}$-meet (the $\leq_{t}$-join) and by $\otimes$ (respectively, by $\oplus$ ) the $\leq_{k}$-meet (the $\leq_{k}$-join) of $\mathcal{B}$. While the meaning of $\wedge$ and $\vee$ corresponds to the standard logical role of these operators, the intuition behind $\otimes$ and $\oplus$ is somewhat less transparent. Fitting ${ }^{9}$ calls them consensus and gullibility operations, respectively, to indicate that $x \otimes y$ is the most information 'agreed' upon by $x$ and $y$, while $x \oplus y$ includes everything accepted by at least one of $x$ and $y$.

We denote by $f$ and $t$ the $\leq_{t^{-}}$extreme elements, and $\perp$, $\top$ denote the $\leq_{k^{-}}$ extreme elements of $\mathcal{B}$. Intuitively, these elements can be perceived as 'false', 'true', 'unknown' (i.e., neither true nor false) and 'contradictory' (both true and false), respectively. Thus, for instance, $f \leq_{t} \perp$ since the 'degree of truth' of a statement which is known to be false is smaller than that of a statement about which there is no information whatsoever. On the other hand, $\perp \leq_{k} f$, since knowing that a statement is false is more informative than knowing nothing at all about it.

Clearly, the more interesting forms of bilattices are those in which the two partial orders are related in one way or another. Below are some common types of such relations:

Definition 2. Let $\mathcal{B}=\left(B, \leq_{t}, \leq_{k}\right)$ be a bilattice.

- $\mathcal{B}$ is called distributive ${ }^{5}$ if all the (twelve) possible distributive laws concerning $\wedge, \vee, \otimes$, and $\oplus$ hold (for instance, $a \wedge(b \oplus c)=(a \wedge b) \oplus(a \wedge c)$ ).

- $\mathcal{B}$ is called interlaced ${ }^{6}$ if each one of $\wedge, \vee, \otimes$, and $\oplus$, is monotonic with respect to both $\leq_{t}$ and $\leq_{k}$ (for instance, if $a \leq_{k} b$ then $a \wedge c \leq_{k} b \wedge c$ ).

- $\mathcal{B}$ is a bilattice with a negation ${ }^{5}$ if there exists a unary operation $\neg$ satisfying, for every $x, y$ in $B,(1) \neg \neg x=x$, (2) if $x \leq_{t} y$ then $\neg x \geq_{t} \neg y$, and (3) if $x \leq_{k} y$ then $\neg x \leq_{k} \neg y$.

- $\mathcal{B}$ is a bilattice with a conflation ${ }^{8}$ if there exists a unary operation - satisfying, for every $x, y$ in $B,(1)--x=x$, (2) if $x \leq_{k} y$ then $-x \geq_{k}-y$, and (3) if $x \leq_{t} y$ then $-x \leq_{t}-y$.

- $\mathcal{B}$ is called classical, ${ }^{15}$ if it has a negation $\neg$ and a conflation - that commute, and for every $x$ in $B$ it holds that $x \vee-\neg x=t$.

Originally, Ginsberg considered bilattices with negations. In this case a negation is an involution with respect to the lattice $\left(B, \leq_{t}\right)$ and an order preserving operation of the lattice $\left(B, \leq_{k}\right)$. Conversely, a conflation is an involution of $\left(B, \leq_{k}\right)$ and an order preserving operation of $\left(B, \leq_{t}\right)$. Following Ginsberg, Fitting introduced the family of interlaced bilattices and showed their usefulness for logic programming (see e.g. Refs. 6,7 ). It is easily verified that distributive bilattices are also interlaced. In the context of fuzzy sets, interlaced bilattices have been considered, e.g., in Ref. 16. 
Example 1. Figure 1 depicts double-Hasse diagrams of a four-valued bilattice $\mathcal{F O U R}$ (after Belnap's four-valued structure ${ }^{17,18}$ ) and a nine-valued bilattice $\mathcal{N} \mathcal{I} \mathcal{N} \mathcal{E}$ that contains, in addition to the four basic elements of $\mathcal{F} \mathcal{O U} \mathcal{R}$, elements for representing true/false default assumptions $(d t, d f)$ for reasoning with lack of information, conflict situations that are 'biased' for true or false $(b t, b f)$ for reasoning with too much information, and a neutral, middle element $m$ (see Ref. 19, 20 for a more detailed discussion on these structures and their applications). Both these bilattices are distributive, and so they are interlaced as well. Note that $\mathcal{F} \mathcal{O U} \mathcal{R}$ is classical, while $\mathcal{N} \mathcal{I} \mathcal{N} \mathcal{E}$ is not. An example of a (distributive) bilattice with an infinite amount of elements is $\left([0,1]^{2}, \leq_{t}, \leq_{k}\right)$, consisting of pairs of elements from the unit interval, with $\leq_{t}$ and $\leq_{k}$ defined as

$$
\begin{aligned}
& \left(x_{1}, x_{2}\right) \leq_{t}\left(y_{1}, y_{2}\right) \Leftrightarrow x_{1} \leq y_{1} \text { and } x_{2} \geq y_{2}, \\
& \left(x_{1}, x_{2}\right) \leq_{k}\left(y_{1}, y_{2}\right) \Leftrightarrow x_{1} \leq y_{1} \text { and } x_{2} \leq y_{2} .
\end{aligned}
$$

It is worth mentioning that in the context of fuzzy set theory, the $\leq_{t}$-ordering of this bilattice has been studied in Refs. 21,22 and its $\leq_{k}$-ordering is considered in Ref. 23.
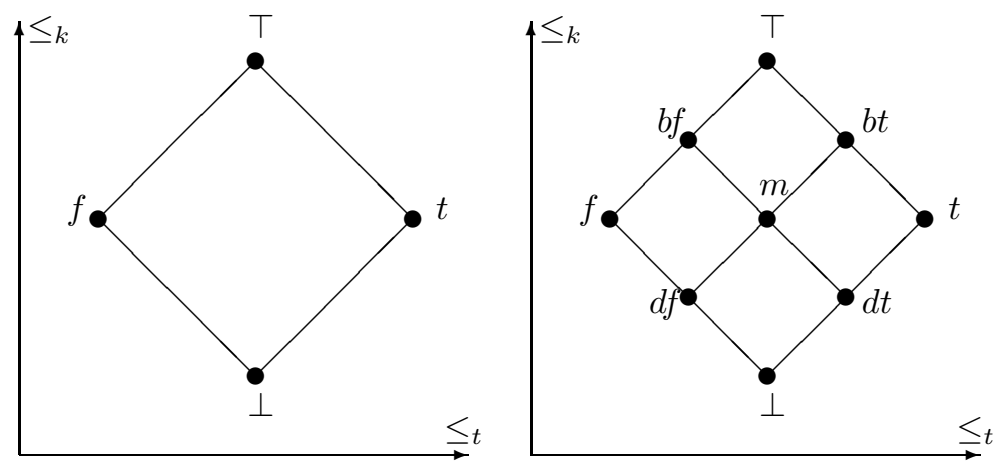

Fig. 1. The bilattices $\mathcal{F O U R}$ and $\mathcal{N} \mathcal{I N E}$.

The following proposition recalls some important properties of bilattices.

\section{Proposition 1.}

- ${ }^{5}$ Let $\mathcal{B}=\left(B, \leq_{t}, \leq_{k}\right)$ be a bilattice with a negation $\neg$. For all $x, y$ in $B$ :
(a) $\neg(x \wedge y)=\neg x \vee \neg y, \neg(x \vee y)=\neg x \wedge \neg y, \neg(x \otimes y)=\neg x \otimes \neg y, \neg(x \oplus y)=$ $\neg x \oplus \neg y$.
(b) $\neg f=t, \neg t=f, \neg \perp=\perp, \neg \top=\top$.

- ${ }^{8}$ Let $\mathcal{B}=\left(B, \leq_{t}, \leq_{k}\right)$ be a bilattice with a conflation - For all $x, y$ in $B$ :
(a) $-(x \wedge y)=-x \wedge-y,-(x \vee y)=-x \vee-y, \quad-(x \otimes y)=-x \oplus-y,-(x \oplus y)=$ $-x \otimes-y$.
(b) $-f=f,-t=t,-\perp=\top,-\top=\perp$.




\subsection{Rectangular bilattices}

Definition 3. Let $\mathcal{L}=\left(L, \leq_{L}\right)$ and $\mathcal{R}=\left(R, \leq_{R}\right)$ be two complete lattices. A rectangular bilattice, shortly rectangle, is a structure $\mathcal{L} \odot \mathcal{R}=\left(L \times R, \leq_{t}, \leq_{k}\right)$, where, for every $x_{1}, y_{1}$ in $L$ and $x_{2}, y_{2}$ in $R$,

$$
\begin{aligned}
& \text { (1) } \quad\left(x_{1}, x_{2}\right) \leq_{t}\left(y_{1}, y_{2}\right) \Leftrightarrow x_{1} \leq_{L} y_{1} \text { and } x_{2} \geq_{R} y_{2}, \\
& \text { (2) }\left(x_{1}, x_{2}\right) \leq_{k}\left(y_{1}, y_{2}\right) \Leftrightarrow x_{1} \leq_{L} y_{1} \text { and } x_{2} \leq_{R} y_{2} .
\end{aligned}
$$

We say that a structure is rectangular if it is isomorphic to a rectangular bilattice. An element $\left(x_{1}, x_{2}\right)$ of a rectangle $\mathcal{L} \odot \mathcal{R}$ may intuitively be understood such that $x_{1}$ represents the amount of belief for some assertion, and $x_{2}$ is the amount of belief against it. In the context of fuzzy sets, this corresponds to Atanassov's theory of intuitionistic fuzzy sets, ${ }^{13}$ which extends standard fuzzy set theory so that any element $u$ in a universe $U$ is assigned not only a membership degree, $\mu_{A}(u)$, but also a non-membership degree $\nu_{A}(u)$, where both degrees are drawn from the unit interval $[0,1]$ and satisfy the condition $\mu_{A}(u)+\nu_{A}(u) \leq 1$. Rectangular bilattices generalize this idea by not imposing the latter condition, by considering arbitrary lattices (not only the unit interval), and by defining the membership function and the non-membership function over potentially different ranges (see also Section 3 below).

Denote the join and meet operations of a complete lattice $\mathcal{L}=\left(L, \leq_{L}\right)$ by $\wedge_{L}$ and $\vee_{L}$, respectively. Then, for every $x_{1}, y_{1}$ in $L$ and $x_{2}, y_{2}$ in $R$, we have

$$
\begin{aligned}
& \left(x_{1}, x_{2}\right) \wedge\left(y_{1}, y_{2}\right)=\left(x_{1} \wedge_{L} y_{1}, x_{2} \vee_{R} y_{2}\right), \\
& \left(x_{1}, x_{2}\right) \vee\left(y_{1}, y_{2}\right)=\left(x_{1} \vee_{L} y_{1}, x_{2} \wedge_{R} y_{2}\right), \\
& \left(x_{1}, x_{2}\right) \otimes\left(y_{1}, y_{2}\right)=\left(x_{1} \wedge_{L} y_{1}, x_{2} \wedge_{R} y_{2}\right), \\
& \left(x_{1}, x_{2}\right) \oplus\left(y_{1}, y_{2}\right)=\left(x_{1} \vee_{L} y_{1}, x_{2} \vee_{R} y_{2}\right),
\end{aligned}
$$

Moreover, denoting $0_{\mathcal{L}}=\inf L$ and $1_{\mathcal{L}}=\sup L$, it holds that

$$
\perp_{\mathcal{L} \odot \mathcal{R}}=\left(0_{\mathcal{L}}, 0_{\mathcal{R}}\right), \quad \top_{\mathcal{L} \odot \mathcal{R}}=\left(1_{\mathcal{L}}, 1_{\mathcal{R}}\right), \quad t_{\mathcal{L} \odot \mathcal{R}}=\left(1_{\mathcal{L}}, 0_{\mathcal{R}}\right), \quad f_{\mathcal{L} \odot \mathcal{R}}=\left(0_{\mathcal{L}}, 1_{\mathcal{R}}\right) .
$$

It is easy to verify that a rectangular bilattice is indeed a bilattice (in the sense of Definition 1). The next proposition summarizes some basic properties of rectangular bilattices and shows their central role in the theory of bilattices:

\section{Proposition 2.}

(a) ${ }^{6}$ Every rectangular bilattice is interlaced.

(b) ${ }^{24}$ Every interlaced bilattice is rectangular.

(c) ${ }^{5}$ If $\mathcal{L}$ and $\mathcal{R}$ are distributive lattices then $\mathcal{L} \odot \mathcal{R}$ is a distributive bilattice.

(d) ${ }^{5,6}$ Every distributive bilattice is isomorphic to $\mathcal{L} \odot \mathcal{R}$ for some distributive lattices $\mathcal{L}$ and $\mathcal{R}$.

In the context of item (b) of the proposition above, it is interesting to note that every interlaced bilattice $\mathcal{B}=\left(B, \leq_{t}, \leq_{k}\right)$ is isomorphic to the structure $\mathcal{L} \odot \mathcal{R}$, 
where $\mathcal{L}=\left(\left\{x \mid x \geq_{t} \perp\right\}, \leq_{k}\right)$ and $\mathcal{R}=\left(\left\{x \mid x \leq_{t} \perp\right\}, \leq_{k}\right)$. These lattices are unique up to an isomorphism (see Ref. 24). The same lattices may be used for item (d) of the proposition above, together with the observation that if $\mathcal{B}$ is a distributive bilattice, then $\mathcal{L}$ and $\mathcal{R}$ are necessarily distributive lattices.

\subsection{Squares and triangles}

An important family of rectangular bilattices are those in which $\mathcal{L}$ and $\mathcal{R}$ coincide. These bilattices are called squares $s^{2,3,21,22}$ and $\mathcal{L} \odot \mathcal{L}$ is abbreviated by $\mathcal{L}^{2}$.

Example 2. The two bilattices shown in Figure 1 above are squares. In fact, $\mathcal{F O U R}$ is isomorphic to $\mathcal{L}_{2}^{2}$, where $\mathcal{L}_{2}=(\{0,1\}, \leq)$, and $\mathcal{N} \mathcal{I N \mathcal { E }}$ is isomorphic to $\mathcal{L}_{3}^{2}$, where $\mathcal{L}_{3}=\left(\left\{0, \frac{1}{2}, 1\right\}, \leq\right)$, with $\leq$ in each case the usual ordering.

Again, it is easy to verify that every square $\mathcal{L}^{2}$ is interlaced, and that it is distributive when $\mathcal{L}$ is distributive. The following proposition shows that the converse is also true.

Proposition 3. ${ }^{24}$ Every interlaced bilattice with a negation is isomorphic to a square, equipped with a negation $\neg$ defined, for every $x, y$ in $L$, by $\neg(x, y)=(y, x)$.

Remark 1. Proposition 3 implies the following two corollaries:

- Since every distributive bilattice is also interlaced, from Proposition 3 it follows that distributive bilattices with a negation are isomorphic to squares.

- By Proposition 2 and Proposition 3, any rectangular bilattice with a negation is isomorphic to a square.

In contrast to the previous proposition, the next one shows that squares are not necessarily associated with classical bilattices (as we have seen, e.g., in the case of $\mathcal{N} \mathcal{I} \mathcal{N})$ :

Proposition 4. Let $\mathcal{L}=\left(L, \leq_{L}\right)$ be a complete lattice with at least 3 elements and let $\sup L=1_{\mathcal{L}}$ and $\inf L=0_{\mathcal{L}}$, such that $1_{\mathcal{L}}$ is join irreducible. ${ }^{2}$ Then $\mathcal{L}^{2}$ is not classical.

Proof. Consider some $x$ in $L$ different from $0_{\mathcal{L}}$ and $1_{\mathcal{L}}$. Then: $\left(0_{\mathcal{L}}, 0_{\mathcal{L}}\right)<_{k}$ $\left(x, 0_{\mathcal{L}}\right)<_{k}\left(1_{\mathcal{L}}, 0_{\mathcal{L}}\right)$. Hence, for any conflation - of $\mathcal{L}^{2},-\left(0_{\mathcal{L}}, 0_{\mathcal{L}}\right)>_{k}-\left(x, 0_{\mathcal{L}}\right)>_{k}$ $-\left(1_{\mathcal{L}}, 0_{\mathcal{L}}\right)$. Thus, $\left(1_{\mathcal{L}}, 1_{\mathcal{L}}\right)>_{k}-\left(x, 0_{\mathcal{L}}\right)>_{k}\left(1_{\mathcal{L}}, 0_{\mathcal{L}}\right)$, and so $-\left(x, 0_{\mathcal{L}}\right)=\left(1_{\mathcal{L}}, y\right)$ for some $y$ in $L \backslash\left\{0_{\mathcal{L}}, 1_{\mathcal{L}}\right\}$. But then, $\left(1_{\mathcal{L}}, 1_{\mathcal{L}}\right)<_{t}\left(1_{\mathcal{L}}, y\right)<_{t}\left(1_{\mathcal{L}}, 0_{\mathcal{L}}\right)$. Hence, for any negation $\neg$ of $\mathcal{L}^{2},\left(1_{\mathcal{L}}, 1_{\mathcal{L}}\right)=\neg\left(1_{\mathcal{L}}, 1_{\mathcal{L}}\right)>_{t} \neg\left(1_{\mathcal{L}}, y\right)>_{t} \neg\left(1_{\mathcal{L}}, 0_{\mathcal{L}}\right)=\left(0_{\mathcal{L}}, 1_{\mathcal{L}}\right)$. Thus, $\neg-\left(x, 0_{\mathcal{L}}\right)=\neg\left(1_{\mathcal{L}}, y\right)=\left(z, 1_{\mathcal{L}}\right)$ for some $z$ in $L \backslash\left\{0_{\mathcal{L}}, 1_{\mathcal{L}}\right\}$. Since $1_{\mathcal{L}}$ is joinirreducible, we obtain $\left(x, 0_{\mathcal{L}}\right) \vee \neg-\left(x, 0_{\mathcal{L}}\right)=\left(x, 0_{\mathcal{L}}\right) \vee\left(z, 1_{\mathcal{L}}\right)=\left(x \vee_{L} z, 0_{\mathcal{L}} \wedge_{L} 1_{\mathcal{L}}\right) \neq$ $\left(1_{\mathcal{L}}, 0_{\mathcal{L}}\right)$. Hence $\mathcal{L}^{2}$ is not classical.

${ }^{2}$ I.e., $x \vee y=1_{\mathcal{L}}$ iff $x=1_{\mathcal{L}}$ or $y=1_{\mathcal{L}}$. 
Corollary 1. Let $\mathcal{L}=\left(L, \leq_{L}\right)$ be a chain with at least 3 elements. Then $\mathcal{L}^{2}$ is not classical.

The following family of related structures is due to Fitting. ${ }^{8}$

Definition 4. Let $\mathcal{L}=\left(L, \leq_{L}\right)$ be a complete lattice, and let $I(L)=\left\{\left[x_{1}, x_{2}\right] \mid\right.$ $\left(x_{1}, x_{2}\right) \in L^{2}$ and $\left.x_{1} \leq_{L} x_{2}\right\}$. A (bilattice-based) triangle is a structure $\mathcal{I}(\mathcal{L})=$ $\left(I(L), \leq_{t}, \leq_{k}\right)$, defined for every $\left[x_{1}, x_{2}\right],\left[y_{1}, y_{2}\right]$ in $I(L)$ as follows:

(1) $\left[x_{1}, x_{2}\right] \leq_{t}\left[y_{1}, y_{2}\right] \Leftrightarrow x_{1} \leq_{L} y_{1}$ and $x_{2} \leq_{L} y_{2}$,

(2) $\left[x_{1}, x_{2}\right] \leq_{k}\left[y_{1}, y_{2}\right] \Leftrightarrow x_{1} \leq_{L} y_{1}$ and $x_{2} \geq_{L} y_{2}$.

Note that, in fact, a triangle $\mathcal{I}(\mathcal{L})$ is not a bilattice, since the substructure $\left(I(L), \leq_{k}\right)$ is not a lattice (the supremum of any two elements does not necessarily exist). Still, triangles are very much in the same spirit as bilattices, since the $\leq_{k^{-}}$ ordering also represents differences in the amount of information that each interval exhibits. For this reason, $\mathcal{I}(\mathcal{L})$ is sometimes called a " $\leq_{k}$-lower pre-bilattice" ${ }^{25}$ or a "weak interlaced bilattice". ${ }^{26}$

Example 3. The triangles $\mathcal{I}\left(\mathcal{L}_{2}\right)$ and $\mathcal{I}\left(\mathcal{L}_{3}\right)$ are shown in Figure 2. It is easy to see that, e.g., $[0,0]$ and $[1,1]$ have no common upper bound with respect to $\leq_{k}$.
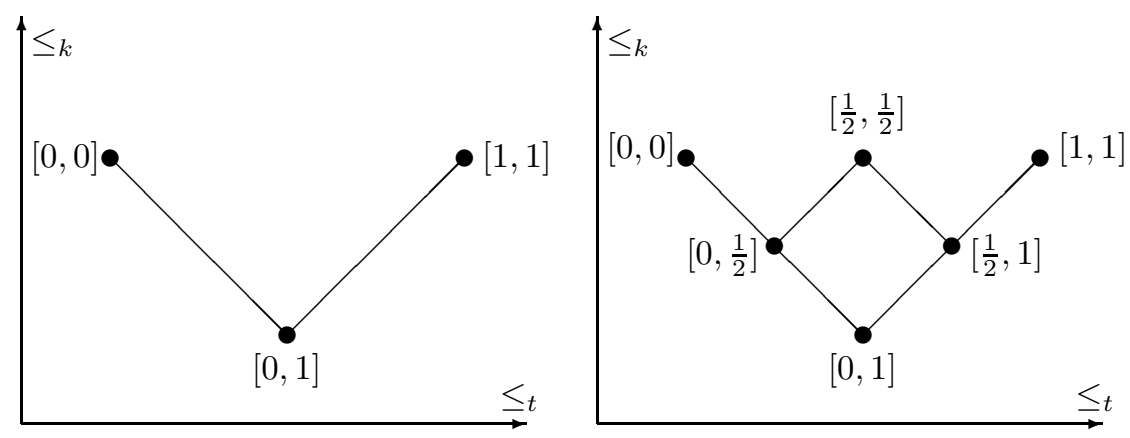

Fig. 2. The triangles $\mathcal{I}\left(\mathcal{L}_{2}\right)$ and $\mathcal{I}\left(\mathcal{L}_{3}\right)$.

Definition 5. ${ }^{8}$ Let $\mathcal{B}=\left(B, \leq_{t}, \leq_{k}\right)$ be a bilattice with a conflation -. An element $x$ in $B$ is called exact w.r.t. this conflation if $x=-x$; it is consistent if $x \leq_{k}-x$.

Intuitively, exact elements exhibit precise information about the underlying assertions, while the consistent ones endorse unambiguous (non-contradictory) evidence about their assertions.

Definition 6. Let - be a conflation of a bilattice $\mathcal{B}=\left(B, \leq_{t}, \leq_{k}\right)$. Denote by $\mathcal{C}^{-}(\mathcal{B})$ the substructure of $\mathcal{B}$ that consists of the consistent elements (w.r.t. - ) of $B$. 
The following proposition relates squares and triangles:

Proposition 5. ${ }^{8}$ Suppose that $\mathcal{L}$ is a complete lattice with an involution $\mathcal{N}$. Then $\mathcal{L}^{2}$ has a conflation $-_{\mathcal{N}}$ that is defined in terms of $\mathcal{N}$, and it holds that $\mathcal{I}(\mathcal{L})$ is isomorphic to $\mathcal{C}^{-\mathcal{N}}\left(\mathcal{L}^{2}\right)$.

In other words, Proposition 5 shows that the triangle $\mathcal{I}(\mathcal{L})$ is isomorphic to the substructure of the square $\mathcal{L}^{2}$, consisting of the consistent elements of $L^{2}$. The isomorphism $f$ between $\mathcal{I}(\mathcal{L})$ and $\mathcal{C}^{-\mathcal{N}}\left(\mathcal{L}^{2}\right)$ for the proposition above is given by $f\left(\left[x_{1}, x_{2}\right]\right)=\left(x_{1}, \mathcal{N}\left(x_{2}\right)\right)$.

In the next section we shall consider the consequences of Proposition 5 in the context of fuzzy sets and some of their generalizations.

\section{Relating Generalized Fuzzy Sets through Bilattices}

In this section we elaborate on the observation given in Refs. 2 and 3, that the bilattice structures considered in the previous section provide an elegant framework for bridging between intuitionistic fuzzy sets (IFSs) and interval-valued fuzzy sets (IVFSs). In particular, the syntactical equivalence of these two commonly encountered extensions of Zadeh's fuzzy sets will be clarified.

The theory of intuitionistic fuzzy sets (IFS) ${ }^{13}$ is an extension of Zadeh's fuzzy set theory, ${ }^{27}$ in which any element $u$ in a universe $U$ is assigned not only a membership degree, $\mu_{A}(u)$, but also a non-membership degree $\nu_{A}(u)$, where both degrees are drawn from the unit interval $[0,1]$. While in fuzzy set theory always $\nu_{A}(u)=1-$ $\mu_{A}(u)$, in IFS theory a weaker constraint is imposed: $\nu_{A}(u) \leq 1-\mu_{A}(u) .{ }^{3}$ IFSs in a universe $U$ can also be regarded as a particular kind of $\mathcal{L}$-fuzzy sets in the sense of Goguen, ${ }^{1}$ i.e. as mappings from $U$ into the complete lattice $\mathcal{L}^{*}$, which is defined as follows:

Definition 7. ${ }^{29}$ Denote by $\mathcal{L}^{*}=\left(L^{*}, \leq_{L^{*}}\right)$, where

- $L^{*}=\left\{\left(x_{1}, x_{2}\right) \mid\left(x_{1}, x_{2}\right) \in[0,1]^{2}\right.$ and $\left.x_{1}+x_{2} \leq 1\right\}$

- $\left(x_{1}, x_{2}\right) \leq_{L^{*}}\left(y_{1}, y_{2}\right)$ iff $x_{1} \leq y_{1}$ and $x_{2} \geq y_{2}$.

Atanassov and Stoeva ${ }^{30}$ introduced the following generalization of the IFS construct, called an intuitionistic $\mathcal{L}$-fuzzy set (ILFS).

Definition 8. Let $\mathcal{L}=\left(L, \leq_{L}\right)$ be a complete lattice with an involution $\mathcal{N}$. An intuitionistic $\mathcal{L}$-fuzzy set in a universe $U$ w.r.t. $\mathcal{N}$ is a mapping $A$ from $U$ to $L^{2}$ such that if $A(u)=\left(x_{1}, x_{2}\right)$ then $x_{1} \leq_{L} \mathcal{N}\left(x_{2}\right)$.

\footnotetext{
${ }^{3}$ The 'intuitionistic' characterizations of this approach should be understood here in a 'broad' sense, as it loosely denies the law of excluded middle (whenever $\mu_{A}(u)+\nu_{A}(u)<1$ ). This approach bears no relationship to the conservative extension of intuitionistic logic, introduced in Ref. 28.
} 
The theory of interval-valued fuzzy sets (IVFS), apparently introduced independently in 1975 by Grattan-Guinness, ${ }^{31}$ Jahn, ${ }^{32}$ Sambuc ${ }^{14}$ and Zadeh, ${ }^{33}$ and later also studied by e.g. Esteva et al., ${ }^{10}$ Gorzałczany, ${ }^{34}$ and Türkşen, ${ }^{35}$ is an alternative method of extending fuzzy set theory, motivated by the need to replace crisp, $[0,1]$ valued membership degrees by intervals in $[0,1]$ that approximate the (unknown) membership degrees. Interval-valued fuzzy sets are also $\mathcal{L}$-fuzzy sets, for which the corresponding lattice is $\mathcal{L}^{I}$, defined as follows:

Definition 9. ${ }^{36}$ Denote by $\mathcal{L}^{I}$ the lattice $\left(L^{I}, \leq_{L^{I}}\right)$, where

- $L^{I}=\left\{\left[x_{1}, x_{2}\right] \mid\left(x_{1}, x_{2}\right) \in[0,1]^{2}\right.$ and $\left.x_{1} \leq x_{2}\right\}$,

- $\left[x_{1}, x_{2}\right] \leq_{L^{I}}\left[y_{1}, y_{2}\right]$ iff $x_{1} \leq y_{1}$ and $x_{2} \leq y_{2}$.

Remark 2. When $\mathcal{L}$ is the unit interval with the usual ordering, $\mathcal{I}(\mathcal{L})$ is a structure that extends the lattice $\mathcal{L}^{I}$ in the sense that $\mathcal{L}^{I}$ is exactly $\left(I([0,1]), \leq_{t}\right)$ and moreover, $\mathcal{I}(\mathcal{L})$ also contains the partially ordered set $\left(I([0,1]), \leq_{k}\right)$ that orders intervals according to their exactness.

Now, in the context of fuzzy sets, Proposition 5 provides a direct relation between IFSs and IVFSs. Moreover,

(a) In case that $L$ is the unit interval, we can define the isomorphism of Proposition 5 by $f\left(\left[x_{1}, x_{2}\right]\right)=\left(x_{1}, 1-x_{2}\right)$, which is exactly the same transformation considered in Ref. 29 for switching between IVFSs and IFSs. Proposition 5 shows that the same transformation is useful not only for relating $\mathcal{L}^{I}$ and $\mathcal{L}^{*}$ (i.e., when the underlying lattice is the unit interval with the standard ordering and involution), but also for any complete lattice with an involution.

(b) Proposition 5 may also serve as a clarification of Atanassov's decision to consider only the 'lower triangle' of $[0,1]^{2}$ (i.e., the elements $\left(x_{1}, x_{2}\right)$ in $[0,1]^{2}$ s.t. $\left.x_{1}+x_{2} \leq 1\right)$ : these are exactly the consistent elements of $[0,1]^{2}$ when the conflation is defined, for every $\left(x_{1}, x_{2}\right)$ in $[0,1]^{2}$, by $-\left(x_{1}, x_{2}\right)=\left(1-x_{2}, 1-x_{1}\right)$. But in that case, it makes sense, and it is probably more intuitive, to work with the lattice $\mathcal{L}^{I}$ from IVFS theory to which $\mathcal{L}^{*}$ is effectively isomorphic by Proposition 5 . The fact that we consider superlattices of $\mathcal{L}^{*}$ (i.e., all the elements in $[0,1]^{2}$ are considered) allows us to introduce elements such as $\left(x_{1}, x_{2}\right)=(1,1)$, in which the membership degree $\left(x_{1}\right)$ and the non-membership degree $\left(x_{2}\right)$ are both maximal. This means that we have a totally inconsistent belief in this case. As an important aspect of fuzzy set theory is reasoning with uncertainty, such values should not be ruled out. ${ }^{4}$

\footnotetext{
${ }^{4}$ Indeed, the need to represent and reason with contradictory information has been widely acknowledged also outside the context of fuzzy set theory (see, e.g., Refs. 37, 38, 39 for some recent collections of papers on this issue). As the level of inconsistency (or the 'amount of contradictory belief') is, without a doubt, a fuzzy notion, the inconsistent elements considered here may be useful for expressing different levels of contradiction (see also Ref. 40).
} 
(c) A largely analogous discussion applies to ILFSs, whose rationale Proposition 5 nicely embodies. Indeed, it is not difficult to see that the condition on $A$ in Definition 8 actually means that $A(u)$ is restricted to be a consistent element of $L$ w.r.t. the conflation $-\mathcal{N}$ which is defined by $-\mathcal{N}\left(x_{1}, x_{2}\right)=\left(\mathcal{N}\left(x_{2}\right), \mathcal{N}\left(x_{1}\right)\right)$, for all $\left(x_{1}, x_{2}\right)$ in $L^{2}$.

(d) Pankowska and Wygralak ${ }^{41}$ introduced a kind of ILFSs based on $([0,1], \leq)$ with an involution $\mathcal{N}_{n}$ defined for any positive real number $n$ by $\mathcal{N}_{n}(x)=$ $\sqrt{[} n] 1-x^{n}$. It is easy to see that when $n$ increases, so does the number of elements $\left(x_{1}, x_{2}\right)$ in $[0,1]^{2}$ for which $x_{1} \leq \mathcal{N}_{n}\left(x_{2}\right)$, or equivalently, $x_{2} \leq \mathcal{N}_{n}\left(x_{1}\right)$. In fact, if $x \neq 1$, then $\lim _{n \rightarrow+\infty} \mathcal{N}_{n}(x)=1$, but when $x=1$, always $\mathcal{N}_{n}(x)=0$. Hence, when $n$ approaches infinity, the set of consistent elements of $[0,1]^{2}$, with respect to the conflation $-\mathcal{N}_{n}$, approaches $[0,1]^{2}$ without the elements $\left(x_{1}, 1\right)$ and $\left(1, x_{2}\right)$, for which $x_{1}, x_{2}>0$. This shows that when $\mathcal{L}=([0,1], \leq)$ it is possible to define a conflation $-\mathcal{N}$ such that $\mathcal{C}^{-\mathcal{N}}\left(\mathcal{L}^{2}\right)$ is arbitrarily close, but not equal, to $\mathcal{L}^{2}$.

(e) Related to the previous item, in Ref. 42, Atanassov introduced a pair of bijections between $L^{*}$ and $[0,1]^{2}$, which at first glance seems to shatter the remarks made in item (b) that the latter is a more expressive structure. It was shown in Ref. 21, however, that these bijections do not preserve order and hence they do not constitute an isomorphism between $\mathcal{L}^{*}$ and $\left([0,1]^{2}, \leq_{t}\right)$, or, for that matter, between $\mathcal{I}(([0,1], \leq))$ and $([0,1], \leq)^{2}$. The following proposition generalizes this result to any complete lattice $\mathcal{L}$ with an involution $\mathcal{N}$.

Proposition 6. For a complete lattice $\mathcal{L}=\left(L, \leq_{L}\right)$ with an involution $\mathcal{N}$, the structures $\mathcal{L}^{2}$ and $\mathcal{C}^{-\mathcal{N}}\left(\mathcal{L}^{2}\right)$ are not isomorphic.

Proof. The proposition simply follows from the fact that $\mathcal{L}^{2}$ is upward-closed (i.e., the supremum of every two elements of $\mathcal{L}^{2}$ is also an element of $\left.\mathcal{L}^{2}\right)$, while $\mathcal{C}^{-\mathcal{N}}\left(\mathcal{L}^{2}\right)$ is not, so these structures cannot be isomorphic. Below we give another proof, which extends that of Ref. 21 to arbitrary lattices.

Assume that there exists an isomorphism $\Theta$ from $\mathcal{L}^{2}$ to $\mathcal{C}^{-\mathcal{N}}\left(\mathcal{L}^{2}\right)$. Since $\Theta$ is $\leq_{t}$-increasing, we easily find that $\Theta\left(0_{\mathcal{L}}, 1_{\mathcal{L}}\right)=\left(0_{\mathcal{L}}, 1_{\mathcal{L}}\right)$ and $\Theta\left(1_{\mathcal{L}}, 0_{\mathcal{L}}\right)=\left(1_{\mathcal{L}}, 0_{\mathcal{L}}\right)$. Similarly, since $\Theta$ is $\leq_{k}$-increasing, $\Theta\left(0_{\mathcal{L}}, 0_{\mathcal{L}}\right)=\left(0_{\mathcal{L}}, 0_{\mathcal{L}}\right)$.

Let $a=\left(a_{1}, a_{2}\right)=\Theta\left(1_{\mathcal{L}}, 1_{\mathcal{L}}\right)$. Since $a$ is consistent, we have that $\left(a_{1}, a_{2}\right) \leq_{k}$ $\left(\mathcal{N}\left(a_{2}\right), \mathcal{N}\left(a_{1}\right)\right)$ and so $a_{2} \leq_{L} \mathcal{N}\left(a_{1}\right)$ or, equivalently, $a_{1} \leq_{L} \mathcal{N}\left(a_{2}\right)$.

If $a_{2}<_{L} \mathcal{N}\left(a_{1}\right)$, then let $b=\left(a_{1}, \mathcal{N}\left(a_{1}\right)\right)$. Clearly, $b$ is consistent and $a<_{k} b$. Since $\Theta^{-1}$ is strictly $\leq_{k}$-increasing, $\left(1_{\mathcal{L}}, 1_{\mathcal{L}}\right)=\Theta^{-1}(a)<_{k} \Theta^{-1}(b)$, which is a contradiction, since $\left(1_{\mathcal{L}}, 1_{\mathcal{L}}\right)$ is the greatest element of $\left(L^{2}, \leq_{k}\right)$.

If $a_{2}=\mathcal{N}\left(a_{1}\right)>_{L} 0_{\mathcal{L}}$, then $a_{1}<_{L} 1_{\mathcal{L}}$, since $\mathcal{N}$ is strictly $\mathcal{L}$-decreasing. It follows that $a=\left(a_{1}, \mathcal{N}\left(a_{1}\right)\right)$ and $\left(1_{\mathcal{L}}, 0_{\mathcal{L}}\right)$ are $\leq_{k}$-incomparable. On the other hand, $\Theta^{-1}\left(1_{\mathcal{L}}, 0_{\mathcal{L}}\right)=\left(1_{\mathcal{L}}, 0_{\mathcal{L}}\right) \leq_{k}\left(1_{\mathcal{L}}, 1_{\mathcal{L}}\right)=\Theta^{-1}(a)$. Since $\Theta$ is $\leq_{k}$-increasing, it follows that $\left(1_{\mathcal{L}}, 0_{\mathcal{L}}\right) \leq_{k} a$, which is a contradiction.

If $a_{2}=\mathcal{N}\left(a_{1}\right)=0_{\mathcal{L}}$, then $a_{1}=1_{\mathcal{L}}$, so $a=\left(1_{\mathcal{L}}, 0_{\mathcal{L}}\right)$ and $\Theta^{-1}(a)=\left(1_{\mathcal{L}}, 0_{\mathcal{L}}\right)$, which is a contradiction. 


\section{Graded (Bilattice-Based) Logical Connectives}

In this section we recall some common extensions to $\mathcal{L}$-fuzzy set theory of the main connectives of classical logic (see, e.g., Ref. 43), and show how they can be related to rectangular bilattices. A similar analysis for triangles and squares is given in Ref. 4.

In what follows, we assume that $\mathcal{L}=\left(L, \leq_{L}\right)$ is a complete lattice such that $0_{\mathcal{L}}=\inf L$ and $1_{\mathcal{L}}=\sup L$. Proofs of the propositions in this section appear in the appendix at the end of the paper.

\subsection{Negation}

Definition 10. A negator on $\mathcal{L}$ is any $\leq_{L}$-reversing mapping $\mathcal{N}: L \rightarrow L$ such that $\mathcal{N}\left(0_{\mathcal{L}}\right)=1_{\mathcal{L}}$ and $\mathcal{N}\left(1_{\mathcal{L}}\right)=0_{\mathcal{L}}$. If for every $x, \mathcal{N}(\mathcal{N}(x))=x$, then $\mathcal{N}$ is an involutive negator on $\mathcal{L}$.

In the context of bilattices, the operation $\neg$ that appears in Definition 2 is an involutive negator on the lattice $\left(B, \leq_{t}\right)$. On a square $\mathcal{L}^{2}$, such an operator may be defined as follows:

$$
\neg\left(x_{1}, x_{2}\right)=\left(x_{2}, x_{1}\right) .
$$

If a bilattice $\mathcal{B}$ has a conflation - , then by its definition this operation is an involutive negator on the lattice $\left(B, \leq_{k}\right)$.

Suppose now that $\mathcal{N}$ is an involutive negator on $\mathcal{L}$, i.e., every $x$ in $L$ has an $\leq_{L}$-involutive element $\mathcal{N}(x)$ in $L$. Then, as we have noted before, a conflation $-_{\mathcal{N}}$ of $\mathcal{L}^{2}$ may be defined by

$$
{ }_{-\mathcal{N}}\left(x_{1}, x_{2}\right)=\left(\mathcal{N}\left(x_{2}\right), \mathcal{N}\left(x_{1}\right)\right) .
$$

In this case, another natural definition of a negator on $\left(L^{2}, \leq_{t}\right)$ is obtained by combining $\neg$ and $-\mathcal{N}$, that is, for every $\left(x_{1}, x_{2}\right)$ in $L^{2}$,

$$
\sim_{\mathcal{N}}\left(x_{1}, x_{2}\right)=\neg-\mathcal{N}\left(x_{1}, x_{2}\right)=\left(\mathcal{N}\left(x_{1}\right), \mathcal{N}\left(x_{2}\right)\right) .{ }^{5}
$$

One might wonder if there exist other 'interesting' negators apart from the prototypical ones described above. In Ref. 21 , for the particular structure $\left([0,1]^{2}, \leq_{t}\right)$, it was shown that all involutive negators can be generated by simple transformations of the two basic choices $\neg$ and $\sim_{\mathcal{N}}$. The next proposition is a generalization of that result to arbitrary rectangular bilattices:

Proposition 7. Let $\mathcal{L}=\left(L, \leq_{L}\right)$ and $\mathcal{R}=\left(R, \leq_{R}\right)$ be chains. A mapping $\mathfrak{N}$ : $L \times R \rightarrow L \times R$ is an involutive negator on $\left(L \times R, \leq_{t}\right)$ iff either there exist two

\footnotetext{
${ }^{5}$ This operator has also been studied by Wagner, ${ }^{44}$ mainly in the context of four-valued logics, and by Doherty, Driankov and Tsoukias ${ }^{45}$ in the framework of DDT-logic (an operational version of Belnap's four-valued logic; ${ }^{17,18}$ see also Ref. 46$)$, where it is simply called 'complement'.
} 
involutive negators $\mathcal{N}_{1}$ and $\mathcal{N}_{2}$ on $\mathcal{L}$ and $\mathcal{R}$ respectively such that, for all $\left(x_{1}, x_{2}\right)$ in $L \times R$,

$$
\mathfrak{N}\left(x_{1}, x_{2}\right)=\left(\mathcal{N}_{1}\left(x_{1}\right), \mathcal{N}_{2}\left(x_{2}\right)\right),
$$

or there exists an isomorphism $\varphi$ between $\mathcal{L}$ and $\mathcal{R}$, such that, for all $\left(x_{1}, x_{2}\right) \in$ $L \times R$,

$$
\mathfrak{N}\left(x_{1}, x_{2}\right)=\left(\varphi^{-1}\left(x_{2}\right), \varphi\left(x_{1}\right)\right) .
$$

If $\mathcal{L}$ and $\mathcal{R}$ are not isomorphic, Equality (2) in Proposition 7 is excluded, and so we have the following corollary:

Corollary 2. Let $\mathcal{L}=\left(L, \leq_{L}\right)$ and $\mathcal{R}=\left(R, \leq_{R}\right)$ be two non-isomorphic chains. A mapping $\mathfrak{N}: L \times R \rightarrow L \times R$ is an involutive negator on $\left(L \times R, \leq_{t}\right)$ iff there exist two involutive negators $\mathcal{N}_{1}$ and $\mathcal{N}_{2}$ on $\mathcal{L}$ and $\mathcal{R}$, respectively, such that for every $\left(x_{1}, x_{2}\right) \in L \times R, \mathfrak{N}\left(x_{1}, x_{2}\right)=\left(\mathcal{N}_{1}\left(x_{1}\right), \mathcal{N}_{2}\left(x_{2}\right)\right)$.

\subsection{Conjunction and disjunction}

Definition 11. A triangular norm (a $t$-norm, for short) on a lattice $\mathcal{L}=\left(L, \leq_{L}\right)$ is a mapping $\mathcal{T}: L \times L \rightarrow L$ that is $\leq_{L}$-increasing in both arguments, commutative, associative, and satisfies, for every $x$ in $L, \mathcal{T}\left(1_{\mathcal{L}}, x\right)=x$.

Definition 12. A triangular conorm (a t-conorm, for short) on $\mathcal{L}=\left(L, \leq_{L}\right)$ is a mapping $\mathcal{S}: L \times L \rightarrow L$ that is $\leq_{L}$-increasing in both arguments, commutative, associative, and satisfies, for every $x$ in $L, \mathcal{S}\left(0_{\mathcal{L}}, x\right)=x$.

Given a bilattice $\mathcal{B}=\left(B, \leq_{t}, \leq_{k}\right)$, its $\leq_{t}$-meet $\wedge$ and $\leq_{k}$-meet $\otimes$ are clearly t-norms on $\left(B, \leq_{t}\right)$ and $\left(B, \leq_{k}\right)$, respectively. Also, the $\leq_{t}$-join $\vee$ and the $\leq_{k}$-join $\oplus$ of $\mathcal{B}$ are t-conorms on $\left(B, \leq_{t}\right)$, and $\left(B, \leq_{k}\right)$, respectively. This implies that for complete lattices $\mathcal{L}=\left(L, \leq_{L}\right)$ and $\mathcal{R}=\left(R, \leq_{R}\right)$ with meets $\wedge_{L}$ and $\wedge_{R}$ and joins $\vee_{L}$ and $\vee_{R}$ respectively, the following operations are t-norms on $\left(L \times R, \leq_{t}\right)$ and $\left(L \times R, \leq_{k}\right)$, respectively: for all $\left(x_{1}, x_{2}\right),\left(y_{1}, y_{2}\right)$ in $L \times R$,

$$
\begin{aligned}
& \mathcal{T}_{\leq_{t}}\left(\left(x_{1}, x_{2}\right),\left(y_{1}, y_{2}\right)\right)=\left(x_{1} \wedge_{L} y_{1}, x_{2} \vee_{R} y_{2}\right), \\
& \mathcal{T}_{\leq_{k}}\left(\left(x_{1}, x_{2}\right),\left(y_{1}, y_{2}\right)\right)=\left(x_{1} \wedge_{L} y_{1}, x_{2} \wedge_{R} y_{2}\right) .
\end{aligned}
$$

Similarly, the operations below are, respectively, t-conorms on $\left(L \times R, \leq_{t}\right)$ and on $\left(L \times R, \leq_{k}\right)$ : for all $\left(x_{1}, x_{2}\right),\left(y_{1}, y_{2}\right)$ in $L \times R$,

$$
\begin{aligned}
& \mathcal{S}_{\leq_{t}}\left(\left(x_{1}, x_{2}\right),\left(y_{1}, y_{2}\right)\right)=\left(x_{1} \vee_{L} y_{1}, x_{2} \wedge_{R} y_{2}\right), \\
& \mathcal{S}_{\leq_{k}}\left(\left(x_{1}, x_{2}\right),\left(y_{1}, y_{2}\right)\right)=\left(x_{1} \vee_{L} y_{1}, x_{2} \vee_{R} y_{2}\right) .
\end{aligned}
$$

It is easy to see that $\mathcal{T}_{\leq_{t}}$ is the $\leq_{t}$-greatest t-norm on $\left(L \times R, \leq_{t}\right)$ and $\mathcal{T}_{\leq_{k}}$ is the $\leq_{k}$-greatest t-norm on $\left(L \times R, \leq_{k}\right)$. Also, it is easy to see that $\mathcal{S}_{\leq_{t}}$ and $\mathcal{S}_{\leq_{k}}$ are, respectively, the $\leq_{t}$-smallest t-conorm on $\left(L \times R, \leq_{t}\right)$ and the $\leq_{k}$-smallest t-conorm on $\left(L \times R, \leq_{k}\right)$. 
As in the case of squares, the $\leq_{k}$-extreme t-(co)norms are definable by the $\leq_{t}$-extreme t-(co)norms and the other way around (see also Ref. 47 ).

Proposition 8. In every rectangle $\mathcal{L} \odot \mathcal{R}$, for all $\left(x_{1}, x_{2}\right)$ and $\left(y_{1}, y_{2}\right)$ in $L \times R$,

$$
\begin{aligned}
& \mathcal{T}_{\leq_{k}}\left(\left(x_{1}, x_{2}\right),\left(y_{1}, y_{2}\right)\right) \\
& =\mathcal{S}_{\leq_{t}}\left(\mathcal{S}_{\leq_{t}}\left(\mathcal{T}_{\leq_{t}}\left(\left(x_{1}, x_{2}\right),\left(0_{\mathcal{L}}, 0_{\mathcal{R}}\right)\right), \mathcal{T}_{\leq_{t}}\left(\left(y_{1}, y_{2}\right),\left(0_{\mathcal{L}}, 0_{\mathcal{R}}\right)\right)\right), \mathcal{T}_{\leq_{t}}\left(\left(x_{1}, x_{2}\right),\left(y_{1}, y_{2}\right)\right)\right), \\
& \mathcal{S}_{\leq_{k}}\left(\left(x_{1}, x_{2}\right),\left(y_{1}, y_{2}\right)\right) \\
& =\mathcal{S}_{\leq_{t}}\left(\mathcal{S}_{\leq_{t}}\left(\mathcal{T}_{\leq_{t}}\left(\left(x_{1}, x_{2}\right),\left(1_{\mathcal{L}}, 1_{\mathcal{R}}\right)\right), \mathcal{T}_{\leq_{t}}\left(\left(y_{1}, y_{2}\right),\left(1_{\mathcal{L}}, 1_{\mathcal{R}}\right)\right)\right), \mathcal{T}_{\leq_{t}}\left(\left(x_{1}, x_{2}\right),\left(y_{1}, y_{2}\right)\right)\right), \\
& \mathcal{T}_{\leq_{t}}\left(\left(x_{1}, x_{2}\right),\left(y_{1}, y_{2}\right)\right) \\
& =\mathcal{S}_{\leq_{k}}\left(\mathcal{S}_{\leq_{k}}\left(\mathcal{T}_{\leq_{k}}\left(\left(x_{1}, x_{2}\right),\left(0_{\mathcal{L}}, 1_{\mathcal{R}}\right)\right), \mathcal{T}_{\leq_{k}}\left(\left(y_{1}, y_{2}\right),\left(0_{\mathcal{L}}, 1_{\mathcal{R}}\right)\right)\right), \mathcal{T}_{\leq_{k}}\left(\left(x_{1}, x_{2}\right),\left(y_{1}, y_{2}\right)\right)\right), \\
& \mathcal{T}_{\leq_{t}}\left(\left(x_{1}, x_{2}\right),\left(y_{1}, y_{2}\right)\right) \\
& =\mathcal{S}_{\leq_{k}}\left(\mathcal{S}_{\leq_{k}}\left(\mathcal{T}_{\leq_{k}}\left(\left(x_{1}, x_{2}\right),\left(1_{\mathcal{L}}, 0_{\mathcal{R}}\right)\right), \mathcal{T}_{\leq_{k}}\left(\left(y_{1}, y_{2}\right),\left(1_{\mathcal{L}}, 0_{\mathcal{R}}\right)\right)\right), \mathcal{T}_{\leq_{k}}\left(\left(x_{1}, x_{2}\right),\left(y_{1}, y_{2}\right)\right)\right) .
\end{aligned}
$$

The definition of $\mathcal{T}_{\leq_{t}}, \mathcal{S}_{\leq_{t}}, \mathcal{T}_{\leq_{k}}$, and $\mathcal{S}_{\leq_{k}}$ is an example of an effective way of generating t-(co)norms on rectangles $\mathcal{L} \odot \mathcal{R}$ by taking advantage of existing connectives on the underlying lattices $\mathcal{L}$ and $\mathcal{R}$. Intuitively, this amounts to a kind of divide-and-conquer strategy, where conjunction and disjunction on $\mathcal{L} \odot \mathcal{R}$ are split up into simpler operations on $\mathcal{L}$ and $\mathcal{R}$. This leads us to define the notion of $(\mathcal{L}, \mathcal{R})$-representability. ${ }^{6}$

Definition 13. Let $\mathcal{L}=\left(L, \leq_{L}\right)$ and $\mathcal{R}=\left(R, \leq_{R}\right)$ be complete lattices. A t-norm $\mathfrak{T}$ on $\left(L \times R, \leq_{t}\right)$ (respectively, a t-conorm $\left.\mathfrak{S}\right)$ is called $(\mathcal{L}, \mathcal{R})$-representable, if there exist a t-norm $\mathcal{T}$ on $\mathcal{L}$ and a t-conorm $\mathcal{S}$ on $\mathcal{R}$ (respectively, a t-conorm $\mathcal{S}^{\prime}$ on $\mathcal{L}$ and a t-norm $\mathcal{T}^{\prime}$ on $\mathcal{R}$ ) such that, for every $\left(x_{1}, x_{2}\right),\left(y_{1}, y_{2}\right)$ in $L \times R$,

$$
\begin{aligned}
& \mathfrak{T}\left(\left(x_{1}, x_{2}\right),\left(y_{1}, y_{2}\right)\right)=\left(\mathcal{T}\left(x_{1}, y_{1}\right), \mathcal{S}\left(x_{2}, y_{2}\right)\right), \\
& \mathfrak{S}\left(\left(x_{1}, x_{2}\right),\left(y_{1}, y_{2}\right)\right)=\left(\mathcal{S}^{\prime}\left(x_{1}, y_{1}\right), \mathcal{T}^{\prime}\left(x_{2}, y_{2}\right)\right) .
\end{aligned}
$$

The operations $\mathcal{T}$ and $\mathcal{S}$ (resp. $\mathcal{S}^{\prime}$ and $\mathcal{T}^{\prime}$ ) are called the representants of $\mathfrak{T}$ (resp. S).

Analogously, $(\mathcal{L}, \mathcal{R})$-representable t- $($ co $)$ norms on $\left(L \times R, \leq_{k}\right)$ can be defined in the obvious way. In the sequel, if the identity of the lattices is clear from the context, we shall simply be speaking about representable t-(co)norms.

The above definition allows a straightforward construction of $\mathrm{t}$-(co)norms by operations that meet Definitions 11 and 12; it suffices to take any t-norm $\mathcal{T}$ on $\mathcal{L}$ and t-conorm $\mathcal{S}$ on $\mathcal{R}$, and to use them as representants in formulas (3) and (4) above. The converse, however, is not true; not any t-(co)norm on $\left(L \times R, \leq_{t}\right)$ can be obtained

\footnotetext{
${ }^{6}$ This definition extends the notion of $t$-representability, introduced in Ref. 48, and the notion of $\mathcal{L}$-representability, introduced in Ref. 4.
} 
by a representation. Define, for instance, the mapping $\mathfrak{T}:(L \times R)^{2} \rightarrow(L \times R)$, given by, for all $x, y$ in $L \times R$,

$$
\mathfrak{T}(x, y)=\left(\inf \left(x_{1}, y_{1}, \sup \left(f\left(x_{2}\right), f\left(y_{2}\right)\right)\right), \sup \left(x_{2}, y_{2}\right)\right),
$$

where $f: R \rightarrow L$ is an arbitrary decreasing mapping, the range of which contains at least two elements, and for which $f\left(0_{\mathcal{R}}\right)=1_{\mathcal{L}}$ and $f(\sup (a, b))=\inf (f(a), f(b))$, for all $a, b$ in $R$ (note that e.g. the mapping $f$ for which $f\left(0_{\mathcal{R}}\right)=1_{\mathcal{L}}$ and $f(x)=0_{\mathcal{L}}$, for all $x \in R \backslash\left\{0_{\mathcal{R}}\right\}$, satisfies these constraints). Then $\mathfrak{T}$ is a t-norm on $\left(L \times R, \leq_{t}\right)$, but it is not $(\mathcal{L}, \mathcal{R})$-representable, since its first component also depends on $x_{2}$ and $y_{2}$. Hence, on any rectangle there exist $t$-norms which are not $(\mathcal{L}, \mathcal{R})$-representable.

Proposition 9. Let $\mathfrak{T}$ (respectively, $\mathfrak{S})$ be an $(\mathcal{L}, \mathcal{R})$-representable $t$-norm (respectively, $t$-conorm) on $\left(L \times R, \leq_{t}\right)$. Then $\mathfrak{T}$ (respectively, $\left.\mathfrak{S}\right)$ is increasing w.r.t. both $\leq_{t}$ and $\leq_{k}$. The same property holds for $(\mathcal{L}, \mathcal{R})$-representable $t$-(co)norms with respect to $\left(L \times R, \leq_{k}\right)$.

When a t-norm is not $(\mathcal{L}, \mathcal{R})$-representable the proposition above is no longer true. Indeed, in Ref. 4 it is shown that the t-norm defined in Formula (5) above is not increasing w.r.t. $\leq_{t}$.

Next we relate t-norms and t-conorms by appropriate negators. A natural way of doing so is to impose de Morgan's laws.

Definition 14. Let $\mathcal{T}$ be a t-norm on $\mathcal{L}$, and let $\mathcal{N}$ be an involutive negator on $\mathcal{L}$. The $\mathcal{N}$-dual of $\mathcal{T}$ is a t-conorm $\mathcal{T}_{\mathcal{N}}^{*}$ on $\mathcal{L}$ defined by, for all $x, y$ in $L$,

$$
\mathcal{T}_{\mathcal{N}}^{*}(x, y)=\mathcal{N}(\mathcal{T}(\mathcal{N}(x), \mathcal{N}(y)))
$$

The $\mathcal{N}$-dual of a given t-conorm is defined as a t-norm on $\mathcal{L}$ in a similar way.

Proposition 10. Let $\mathcal{L}$ and $\mathcal{R}$ be two chains, $\mathfrak{T}$ a $(\mathcal{L}, \mathcal{R})$-representable $t$-norm on $\left(L \times R, \leq_{t}\right)$ with representants $\mathcal{T}$ and $\mathcal{S}$, and $\mathfrak{N}$ an involutive negator on $L \times R$. If $\mathfrak{N}\left(0_{\mathcal{L}}, 0_{\mathcal{R}}\right)=\left(1_{\mathcal{L}}, 1_{\mathcal{R}}\right)$, then the $\mathfrak{N}$-dual $t$-conorm $\mathfrak{T}_{\mathfrak{N}}^{*}$ of $\mathfrak{T}$ is given by, for all $x, y$ in $L \times R$,

$$
\mathfrak{T}_{\mathfrak{N}}^{*}(x, y)=\left(\mathcal{T}_{\mathcal{N}_{1}}^{*}\left(x_{1}, y_{1}\right), \mathcal{S}_{\mathcal{N}_{2}}^{*}\left(x_{2}, y_{2}\right)\right),
$$

where $\mathcal{N}_{1}$ and $\mathcal{N}_{2}$ are the representants of $\mathfrak{N}$ (see Proposition 7 ).

If, on the other hand, $\mathfrak{N}\left(0_{\mathcal{L}}, 0_{\mathcal{R}}\right)=\left(0_{\mathcal{L}}, 0_{\mathcal{R}}\right)$, then the $\mathfrak{N}$-dual $t$-conorm $\mathfrak{T}_{\mathfrak{N}}^{*}$ of $\mathfrak{T}$ is given by, for all $x, y$ in $L \times R$,

$$
\mathfrak{T}_{\mathfrak{N}}^{*}(x, y)=\left(\mathcal{S}_{\varphi}\left(x_{1}, y_{1}\right), \mathcal{T}_{\varphi^{-1}}\left(x_{2}, y_{2}\right)\right),
$$

where $\varphi$ is an isomorphism between $\mathcal{L}$ and $\mathcal{R}, \mathcal{S}_{\varphi}$ denotes the $\varphi$-transform of $\mathcal{S}$ defined, for all $x_{1}, y_{1}$ in $L$, by $\mathcal{S}_{\varphi}\left(x_{1}, y_{1}\right)=\varphi^{-1}\left(\mathcal{S}\left(\varphi\left(x_{1}\right), \varphi\left(y_{1}\right)\right)\right)$, and $\mathcal{T}_{\varphi^{-1}}$ is the $\varphi^{-1}$-transform of $\mathcal{T}$, is defined in a similar way. 
As it can be verified from the proof of Proposition 7 (see the appendix), when $\mathcal{L}$ and $\mathcal{R}$ are not isomorphic, the second part of Proposition 10 is excluded, and so we have the following corollary (cf. Corollary 2):

Corollary 3. Let $\mathcal{L}$ and $\mathcal{R}$ be non-isomorphic chains, $\mathfrak{T}$ a $(\mathcal{L}, \mathcal{R})$-representable t-norm on $\left(L \times R, \leq_{t}\right)$ with representants $\mathcal{T}$ and $\mathcal{S}$, and $\mathfrak{N}$ an involutive negator on $L \times R$. Then the $\mathfrak{N}$-dual $t$-conorm $\mathfrak{T}_{\mathfrak{N}}^{*}$ of $\mathfrak{T}$ is given by, for all $x, y$ in $L \times R$,

$$
\mathfrak{T}_{\mathfrak{N}}^{*}(x, y)=\left(\mathcal{T}_{\mathcal{N}_{1}}^{*}\left(x_{1}, y_{1}\right), \mathcal{S}_{\mathcal{N}_{2}}^{*}\left(x_{2}, y_{2}\right)\right),
$$

where $\mathcal{N}_{1}$ and $\mathcal{N}_{2}$ are the representants of $\mathfrak{N}$.

From Proposition 10 it also follows that the dual t-conorm of a t-representable t-norm is also t-representable. If the involved involutive negator maps $\left(0_{\mathcal{L}}, 0_{\mathcal{R}}\right)$ to $\left(1_{\mathcal{L}}, 1_{\mathcal{L}}\right)$, then the first component of the dual t-conorm is obtained using only operations from $\mathcal{L}$, and similarly for the second component, so the dual t-conorm is obtained by calculating the dual operation of each component. On the other hand, if the negator maps $\left(0_{\mathcal{L}}, 0_{\mathcal{R}}\right)$ to $\left(0_{\mathcal{L}}, 0_{\mathcal{R}}\right)$, then the first component of the dual t-conorm is obtained using an operation from $\mathcal{R}$, and similarly for the second component. Similar properties holds for the dual t-norm of a t-representable tconorm.

\subsection{Implication}

Definition 15. An implicator on a lattice $\mathcal{L}=\left(L, \leq_{L}\right)$ is a mapping $\mathcal{I}: L \times L \rightarrow$ $L$ that is $\leq_{L}$-increasing in its first component and $\leq_{L}$-decreasing in its second component, and for which $\mathcal{I}\left(0_{\mathcal{L}}, 0_{\mathcal{L}}\right)=1_{\mathcal{L}}, \mathcal{I}\left(1_{\mathcal{L}}, 0_{\mathcal{L}}\right)=0_{\mathcal{L}}, \mathcal{I}\left(0_{\mathcal{L}}, 1_{\mathcal{L}}\right)=1_{\mathcal{L}}$, and $\mathcal{I}\left(1_{\mathcal{L}}, 1_{\mathcal{L}}\right)=1_{\mathcal{L}}$

Given a t-norm $\mathcal{T}$ and an implicator $\mathcal{I}$ on $\mathcal{L}$, it is usual to require the soundness of fuzzy modus ponens, i.e., for every $a, b, x, y$ in $L$, it should hold that if $a \leq_{L} x$ and $b \leq_{L} \mathcal{I}(x, y)$, then $\mathcal{T}(a, b) \leq_{L} y$. In particular, therefore, if for some $z$ in $L$, $z \leq_{L} \mathcal{I}(x, y)$ then $\mathcal{T}(x, z) \leq_{L} y$. On the other hand, to allow $\mathcal{I}(x, y)$ to be as large as possible, one would like to require the converse, that $\mathcal{T}(x, z) \leq_{L} y$ implies $z \leq_{L} \mathcal{I}(x, y)$. Eventually, then,

$$
\mathcal{T}(x, z) \leq_{L} y \Leftrightarrow z \leq_{L} \mathcal{I}(x, y)
$$

a condition which is known also as the residuation principle. It is well-known (see e.g. Refs. 49, 50) that (6) holds if and only if $\mathcal{T}$ satisfies, for any $x$ in $L$ and any subset $Y$ of $L, \mathcal{T}\left(x, \sup _{y \in Y} y\right)=\sup _{y \in Y} \mathcal{T}(x, y)$, and if $\mathcal{I}$ is equal to the residuum $\mathcal{I}_{\mathcal{T}}$ of $\mathcal{T}$, defined as follows.

Definition 16. Let $\mathcal{T}$ be a t-norm on $\mathcal{L}$. An $R$-implicator $\mathcal{I}_{\mathcal{T}}$ (the residuum of $\mathcal{T}$ ) is defined, for every $x, y$ in $L$, by $\mathcal{I}_{\mathcal{T}}(x, y)=\sup \left\{z \in L \mid \mathcal{T}(x, z) \leq_{L} y\right\}$. 
In the sequel, we will say that a t-norm $\mathcal{T}$ satisfies the residuation principle if (6) holds for $\mathcal{T}$ together with $\mathcal{I}=\mathcal{I}_{\mathcal{T}}$. The following proposition will be important for our further exposition.

Proposition 11. Let $\mathcal{L}=\left(L, \leq_{L}\right)$ and $\mathcal{R}=\left(R, \leq_{R}\right)$ be complete lattices and $\mathcal{T}$ a t-norm on $\left(L \times R, \leq_{t}\right)$ satisfying the residuation principle. Then $\mathcal{T}$ is $(\mathcal{L}, \mathcal{R})$ representable.

Another definition of a family of implicators is motivated by the classical definition of the material implication $x \rightarrow y$ as $\neg x \vee y$.

Definition 17. Let $\mathcal{S}$ be a t-conorm and $\mathcal{N}$ a negator on $\mathcal{L}$. The $S$-implicator $\mathcal{I}_{\mathcal{S}, \mathcal{N}}$ (generated by $\mathcal{S}$ and $\mathcal{N}$ ) is defined for every $x, y$ in $L$ by $\mathcal{I}_{\mathcal{S}, \mathcal{N}}(x, y)=\mathcal{S}(\mathcal{N}(x), y)$.

It is easy to verify that each R-implicator and each S-implicator is in particular an implicator. Moreover, these definitions reveal that very often implicators are linked to "simpler" connectives. Also, we can exploit the classical equivalence between the formulas $x \rightarrow f$ and $\neg x$, to define the following special kind of negators on $\mathcal{L}$.

Definition 18. Let $\mathcal{L}=(L, \leq)$ be a complete lattice with an implicator $\mathcal{I}$. The induced negator of $\mathcal{I}$ is a mapping $\mathcal{N}_{\mathcal{I}}$, defined for every $x$ in $L$ by $\mathcal{N}_{\mathcal{I}}(x)=\mathcal{I}\left(x, 0_{\mathcal{L}}\right)$.

Examples of all the above operations on bilattice-based rectangles are thus easy to generate using the constructs introduced in the previous sections. As an illustration, and in view of its importance for the sequel, the following proposition derives an explicit representation of $\mathrm{R}$-implicators of $(\mathcal{L}, \mathcal{R})$-representable t-norms on $\left(L \times R, \leq_{t}\right)$.

Proposition 12. Let $\mathcal{L}=\left(L, \leq_{L}\right)$ and $\mathcal{R}=\left(R, \leq_{R}\right)$ be complete lattices and let $\mathfrak{T}$ be an $(\mathcal{L}, \mathcal{R})$-representable $t$-norm on $\left(L \times R, \leq_{t}\right)$ with representants $\mathcal{T}$ and $\mathcal{S}$, where $\mathcal{S}$ is the $\mathcal{N}$-dual t-conorm of a t-norm $\mathcal{T}^{\prime}$ for some involutive negator $\mathcal{N}$ on $\mathcal{R}$. Then, for every $\left(x_{1}, x_{2}\right),\left(y_{1}, y_{2}\right)$ in $L \times R$,

$$
\mathfrak{I}_{\mathfrak{T}}\left(\left(x_{1}, x_{2}\right),\left(y_{1}, y_{2}\right)\right)=\left(\mathcal{I}_{\mathcal{T}}\left(x_{1}, y_{1}\right), \mathcal{N}\left(\mathcal{I}_{\mathcal{T}},\left(\mathcal{N}\left(x_{2}\right), \mathcal{N}\left(y_{2}\right)\right)\right)\right) .
$$

The question of implicator classification, i.e., which particular instance to use in which case, has received a considerable attention in the literature. A comprehensive account for the situation in the lattice $\left(I([0,1]), \leq_{t}\right)$ appears in Ref. 36, and $\left([0,1]^{2}, \leq_{t}\right)$ is examined in Refs. 21 and 22. In Ref. 4 the relationship between bilattice-based squares, triangles and MV-algebras is discussed. We now relate rectangular bilattices to MV-algebras.

Definition 19. ${ }^{50,51}$ Let $\mathcal{L}=\left(L, \leq_{L}\right)$ be a complete lattice, and let $\mathcal{T}$ be a t-norm on $\mathcal{L}$ that satisfies the residuation principle (Equation (6)) and such that, for every $x, y$ in $L$,

$$
\mathcal{I}_{\mathcal{T}}\left(\mathcal{I}_{\mathcal{T}}(x, y), y\right)=\mathcal{I}_{\mathcal{T}}\left(\mathcal{I}_{\mathcal{T}}(y, x), x\right)=x \vee_{L} y
$$


Then $\left(L, \leq_{L}, \mathcal{T}\right)$ is called an $M V$-algebra

In an MV-algebra $\left(L, \leq_{L}, \mathcal{T}\right)$, the mappings $\mathcal{I}_{\mathcal{T}}$ and $\mathcal{I}_{\mathcal{S}, \mathcal{N}}$, where $\mathcal{N}=\mathcal{N}_{\mathcal{I}_{\mathcal{T}}}$ and $\mathcal{S}$ is the $\mathcal{N}$-dual of $\mathcal{T}$, are identical. This follows from a result of Jenei, ${ }^{52}$ who proves this identity in general for Girard monoids, of which MV-algebras are a specific case. In Ref. 4 it is shown that the converse is not true in general, that is: coinciding Rand S-implicators would not necessarily lead to an MV-algebra.

A necessary and sufficient condition for having coinciding R- and S-implicators in arbitrary rectangles, is given in the following proposition.

Proposition 13. Let $\mathcal{L}=\left(L, \leq_{L}\right)$ and $\mathcal{R}=\left(R, \leq_{R}\right)$ be complete lattices, and let $\mathfrak{T}$ be a t-norm on $\left(L \times R, \leq_{t}\right)$. Then the $R$-implicator $\mathfrak{I}_{\mathfrak{T}}$ of $\mathfrak{T}$ on $\left(L \times R, \leq_{t}\right)$ is equal to the $S$-implicator $\mathfrak{I}_{\mathfrak{S}, \mathfrak{N}}$ generated by an involutive negator $\mathfrak{N}$ on $\left(L \times R, \leq_{t}\right)$ and the $\mathfrak{N}$-dual $t$-conorm $\mathfrak{S}$ of $\mathfrak{T}$, if and only if there exist $t$-norms $\mathcal{T}$ and $\mathcal{T}^{\prime}$ on $\mathcal{L}$ and $\mathcal{R}$ respectively for which $\mathcal{I}_{\mathcal{T}}=\mathcal{I}_{\mathcal{S}, \mathcal{N}}$ and $\mathcal{I}_{\mathcal{T}^{\prime}}=\mathcal{I}_{\mathcal{S}^{\prime}, \mathcal{N}^{\prime}}$, where $\mathcal{N}$ and $\mathcal{N}^{\prime}$ are involutive negators on $\mathcal{L}$ and $\mathcal{R}$ respectively and where $\mathcal{S}$ (respectively $\mathcal{S}^{\prime}$ ) is the $\mathcal{N}$-dual (respectively $\mathcal{N}^{\prime}$-dual) t-conorm of $\mathcal{T}$ (respectively $\mathcal{T}^{\prime}$ ) such that $\mathfrak{T}$ is $(\mathcal{L}, \mathcal{R})$-representable with representants $\mathcal{T}$ and $\mathcal{S}^{\prime}$.

Proposition 14. Let $\mathcal{L}=\left(L, \leq_{L}\right)$ and $\mathcal{R}=\left(R, \leq_{R}\right)$ be complete lattices, and let $\mathfrak{T}$ be a t-norm on $\left(L \times R, \leq_{t}\right)$. Then $\left(L \times R, \leq_{t}, \mathfrak{T}\right)$ is an MV-algebra if and only if there exist $t$-norms $\mathcal{T}$ and $\mathcal{T}^{\prime}$ on $\mathcal{L}$ and $\mathcal{R}$ respectively such that $\left(L, \leq_{L}, \mathcal{T}\right)$ and $\left(R, \leq_{R}, \mathcal{T}^{\prime}\right)$ are both $M V$-algebras, and such that $\mathfrak{T}$ is $(\mathcal{L}, \mathcal{R})$-representable with representants $\mathcal{T}$ and $\mathcal{S}$, where $\mathcal{S}$ is the $\mathcal{N}$-dual t-conorm of a t-norm $\mathcal{T}^{\prime}$ for some involutive negator $\mathcal{N}$.

\section{Modeling Imprecise Preference Information}

The purpose of this section is to consider the application potential of our framework. We show it in the context of preference modeling.

The notion of preference is common in various contexts involving decision or choice. Preference modeling provides declarative means for choosing among alternatives, including different solutions to problems, answers to database queries, decisions of a computational agent, etc. This topic is gaining increasing attention in diverse areas of artificial intelligence such as nonmonotonic reasoning, qualitative decision theory, configuration, and AI planning. More recently, preference modeling has also been used in constraint satisfaction and constraint programming, for treating soft constraints, for describing search heuristics, and for reducing search effort (see, e.g. Refs. 53 and 54 for recent collections of papers on these topics).

Conventional preference modeling (see e.g. Ref. 55) is centered on the notion of classical preference structures $\langle P, I, R\rangle$, consisting of three fundamental binary relations (strict preference $P$, indifference $I$, and incomparability $R$ ) that may hold among the alternatives; usually the evidence in favour of these relations is captured by a so-called outranking relation $S$ that describes, for each couple $(u, v)$ of alternatives, whether $u$ is (known to be) at least as good as $v$. In practice, it is common 
to encounter situations where these relationships hold up to a certain degree, which gives rise to the study of fuzzy preference structures (see e.g. Refs. 56, 57, 58).

Fuzziness, however, cannot adequately cover all the imperfections inherent to real-life data, since the 'one-dimensional' measurements induced by the ordering of membership degrees in fuzzy sets have difficulties coping with information-deficient data. As Tsoukiàs and Vincke noted in Ref. 59, fuzzy sets and logic per se do not provide " a clear distinction between situations where the information is missing, not satisfactory and situations in which the information is too rich, contradictory, conflictual, ambiguous". Indeed, stating that $P(u, v)=0$ may either mean that $u$ (definitely) is not preferred to $v$, or simply that there is no information to establish a preference of $u$ over $v$, and there is no unambiguous way for a decision maker to distinguish between the two situations. For this reason, several researchers have considered more elaborate means of eliciting and representing preferences. In particular, in a number of papers (e.g. Refs. 60, 61, 62, 63, 59), the use of a four-valued logic called DDT (derived from Belnap's logic $\mathcal{F} \mathcal{O U} \mathcal{R}^{17,18}$ ) and some of its graded extensions has been advocated as a means of dealing preference modeling under incomplete and/or conflicting information. In all of the mentioned papers, bilattice theory per se plays only a subservient role as the convenient 'language' for modeling positive and negative preference arguments separately, and for representing the associated epistemic states of truth, falsity, ignorance and contradiction. As a consequence, these approaches have to incorporate additional conventions and 'tricks' into their modus operandi, making the results less intuitive and/or tedious.

By contrast, the aim of this section is to demonstrate and exploit the full expressive power of rectangular bilattices, and of squares in particular, for encoding not just the problem statement for preference modeling, but also its generic solution strategy. As such, the material presented in what follows is not a 'new' approach to preference modeling, but rather a clarification, simplification and streamlining of existing ones.

\subsection{Encoding the evidence}

The problem at hand is that of ranking a (finite) set $U$ of alternatives from the best to the worst, with respect to a number of given criteria. In order to do this, we assume that partial information is available regarding the pairwise comparison of alternatives. In binary preference modeling, it is common to express such information by means of a two-valued outranking relation $S$ in $U$ (see e.g. Ref. 55), where $S(u, v)=1$ is read as "(there is evidence that) $u$ is at least as good as $v$ ". Such an approach can be criticized for lack of expressivity, since explicit evidence that $u$ is not at least as good as $v$ could only be captured by imposing $S(v, u)=1{ }^{7}$ Yet, as Fortemps and Słowiński argue in Ref. 60, arguments in disfavour of a sentence are not necessarily identical to arguments in favour of the opposite sentence!

\footnotetext{
${ }^{7}$ Note that $S(u, v)=0$ means that there is no evidence that $u$ is at least as good as $v$, which is obviously different than claiming that $u$ is not at least as good as $v$.
} 
For this reason, in Ref. 59 Tsoukiàs and Vincke propose to distinguish between positive and negative arguments regarding the claim ' $u$ is at least as good as $v$ ' $(u \geq v$, for short). Essentially, this amounts to defining the outranking relation $S$ as a mapping from $U^{2}$ to $\{0,1\}^{2}$, where the value of the first (respectively, the second) component of $S(u, v)$ reveals the presence of arguments in favour (respectively, in disfavour) of $u \geq v$. Clearly, this intuition fits our framework, and Belnap's square $\mathcal{F O U R}$ can be used to endow $\{0,1\}^{2}$ with an attractive epistemic structure in terms of truth-hood (the $\leq_{t}$-ordering: from only evidence against, to only evidence for the claim) and of available information (the $\leq_{k}$-ordering: from ignorance to conflict).

Definition 20. For ease of notation, in what follows we shall abbreviate $T$ for $(1,0), \mathrm{F}$ for $(0,1), \mathbf{U}$ for $(0,0)$, and $\mathrm{K}$ for $(1,1)$, to be read as true, false, unknown and contradiction, respectively.

Of course, nothing stands in the way of generalizing this framework by allowing for graded evidence. For instance, in Ref. 60 and 61 the square induced by the unit interval $\mathcal{L}=([0,1], \leq)$ was investigated. In general, $S$ can be a mapping from $U^{2}$ to some rectangular bilattice $\mathcal{L} \odot \mathcal{R}$, reflecting that positive and negative arguments may be evaluated according to two different scales.

\subsection{Representing the preferences}

Once the various outranking arguments have been provided, the objective then is to present the decision maker with as close to reality and transparent as possible a rendering of the actual state of affairs. In conventional preference modeling (i.e., when $S(u, v) \in\{0,1\}$ ), a 'decision' concerning two alternatives $u$ and $v$ can take four forms:

(1) $u$ is (strictly) preferred over $v$ if $S(u, v)=1$ and $S(v, u)=0$,

(2) $v$ is (strictly) preferred over $u$ if $S(u, v)=0$ and $S(v, u)=1$,

(3) $u$ and $v$ are indifferent if $S(u, v)=1$ and $S(v, u)=1$,

(4) $u$ and $v$ are incomparable if $S(u, v)=0$ and $S(v, u)=0$.

Evidently, all possible situations are covered in this way. Accordingly, one can build three binary relations $P$ (strict preference, corresponding to cases 1 and 2 ), $I$ (indifference, corresponding to case 3 ) and $R$ (incomparability, corresponding to case 4), such that $U^{2}=P \cup P^{-1} \cup I \cup R$. It is also said that $\langle P, I, R\rangle$ is a classical preference structure; it is easy to see that it determines $S$ unequivocally, and vice versa ${ }^{8}$; weakened versions emerge when $S$ becomes a fuzzy relation, a theme explored in, e.g., Refs. 56, 57, 58. In what follows, we study the bilattice-valued generalizations of this framework.

${ }^{8}$ Note that $S=P \cup I$. 


\section{A crisp four-valued approach}

Let first $S$ be a mapping from $U^{2}$ to $\{0,1\}^{2}$. Each couple of alternatives $(u, v)$ corresponds to a couple $(S(u, v), S(v, u))$ in $\left(\{0,1\}^{2}\right)^{2}$. For notational ease, and in order to enhance the clarity of the exposition, we shall abbreviate these couples by simply juxtaposing the two letters corresponding to their evaluations. For instance, FK represents the element $((0,1),(1,1))$ that exhibits a situation in which there are only negative arguments for $u \geq v$ and conflicting (both positive and negative) arguments for $v \geq u$.

Remark 3. In Refs. 62, 63, 59, essentially the same representation, albeit in a more complicated form, is obtained by defining, for every $u, v$ in $U$,

$$
\begin{aligned}
\Delta S(u, v) & =1 \Leftrightarrow S(u, v)=(1, x) \text { for some } x \text { in }\{0,1\}, \\
\Delta \neg S(u, v) & =1 \Leftrightarrow S(u, v)=(x, 1) \text { for some } x \text { in }\{0,1\},
\end{aligned}
$$

read as, "there is presence of truth in saying that $u$ is at least as good as $v$ " and "there is presence of truth in saying that $u$ is not at least as good as $v$ ", respectively. Consequently the so-called true, false, contradictory and unknown extensions ${ }^{9}$ of $S(u, v)$ are defined by, respectively,

$$
\begin{aligned}
& \mathbf{T} S(u, v)=1 \Leftrightarrow \Delta S(u, v)=1 \text { and } \Delta \neg S(u, v)=0, \\
& \mathbf{F} S(u, v)=1 \Leftrightarrow \Delta S(u, v)=0 \text { and } \Delta \neg S(u, v)=1, \\
& \mathbf{U} S(u, v)=1 \Leftrightarrow \Delta S(u, v)=0 \text { and } \Delta \neg S(u, v)=0, \\
& \mathbf{K} S(u, v)=1 \Leftrightarrow \Delta S(u, v)=1 \text { and } \Delta \neg S(u, v)=1 .
\end{aligned}
$$

In our notations FK denotes the case where $\mathbf{F} S(u, v)=1$ and $\mathbf{K} S(v, u)=1$.

Thus, a decision maker is confronted with any of sixteen (instead of four) possible situations involving the alternatives $u$ and $v$. As the prime determination is to try to rank the alternatives, it is worthwhile to endow those various situations with some meaningful structure, and it turns out that bilattices can go a long way in doing just that.

Indeed, starting from the $\leq_{t}$-ordering on $\mathcal{F O U} \mathcal{R}$, we can construct a bilatticebased square on top of $\left(\{0,1\}^{2}\right)^{2}$ with the following two orderings:

- $\left(x_{1}, x_{2}\right) \leq_{t}\left(y_{1}, y_{2}\right) \Leftrightarrow x_{1} \leq_{t} y_{1}$ and $x_{2} \geq_{t} y_{2}$

Intuitively, if $\left(x_{1}, x_{2}\right)=(S(u, v), S(v, u))$ and $\left(y_{1}, y_{2}\right)=\left(S\left(u^{\prime}, v^{\prime}\right), S\left(v^{\prime}, u^{\prime}\right)\right)$, then $\left(x_{1}, x_{2}\right) \leq_{t}\left(y_{1}, y_{2}\right)$ expresses that the extent to which $u$ is preferred over $v$ is less than the extent to which $u^{\prime}$ is preferred over $v^{\prime}$. The smallest element is $\mathrm{FT}$ (it is not true that $u \geq v$, while it is true that $u \leq v$ ) and the biggest one is $\operatorname{TF}(u \geq v$ and not $v \geq u)$.

- $\left(x_{1}, x_{2}\right) \leq_{k}\left(y_{1}, y_{2}\right) \Leftrightarrow x_{1} \leq_{t} y_{1}$ and $x_{2} \leq_{t} y_{2}$

${ }^{9}$ These are actually two-valued predicates. In Ref. 60 T, F, U and $\mathbf{K}$ are called 'strong unary operators'. 
This ordering ranges between a state of incomparability (FF) and the one of indifference (TT).

Starting from the $\leq_{k}$-ordering on $\mathcal{F O U \mathcal { R }}$ we can define two other orderings on $\left(\{0,1\}^{2}\right)^{2}$ as follows:

- $\left(x_{1}, x_{2}\right) \leq_{t}^{\prime}\left(y_{1}, y_{2}\right) \Leftrightarrow x_{1} \leq_{k} y_{1}$ and $x_{2} \geq_{k} y_{2}$.

Intuitively, if $\left(x_{1}, x_{2}\right)=(S(u, v), S(v, u))$ and $\left(y_{1}, y_{2}\right)=\left(S\left(u^{\prime}, v^{\prime}\right), S\left(v^{\prime}, u^{\prime}\right)\right)$, then $x_{1} \leq_{k} y_{1}$ means that we know less about $u \geq v$ than about $u^{\prime} \geq v^{\prime}$, and $x_{2} \geq_{k} y_{2}$ means that we know more about $u \leq v$ than about $u^{\prime} \leq v^{\prime}$. So, the bigger $\left(x_{1}, x_{2}\right)$ according to this ordering, the more we know about $u \geq v$ and the less we know about $u \leq v$.

- $\left(x_{1}, x_{2}\right) \leq_{k}^{\prime}\left(y_{1}, y_{2}\right) \Leftrightarrow x_{1} \leq_{k} y_{1}$ and $x_{2} \leq_{k} y_{2}$.

This ordering marks the amount of information at our disposition: from a shortage of information (UU) to an excess (KK).

Remark 4. In Refs. 63, 59, the authors present a dictionary-style solution to discriminate among the sixteen states, giving concrete names and explanations to each one of them. For instance, TF is called 'strict preference of $u$ over $v$ ', KF in their terms is 'weak preference of $u$ over $v$ ', etc. This approach, apart from being tedious, is also misleading. As an example, in their approach (as in ours) FF means that $u$ and $v$ are incomparable, whereas UU is read as " $u$ and $v$ are semi incomparable", and FU as " $u$ and $v$ are weakly incomparable". Such terminology implies an inaccurate description of the state of affairs, since

a) the element UU bears no mark of incomparability whatsoever, and

b) referring to $\leq_{k}$, the elements UF, FK and KF could claim the status of representing 'weak incomparability' with just as much justification as FU.

By contrast, the four order relations considered above serve to discriminate much more naturally, and without bias, among the sixteen states, positioning each state along four scales of measurement.

\section{Extensions to arbitrary (possibly continuous) rectangular bilattices}

Another important advantage of our approach is that it can be straightforwardly generalized to graded evidence without the need for additional parameters. Indeed, the four orderings $\leq_{t}, \leq_{k}, \leq_{t}^{\prime}$, and $\leq_{k}^{\prime}$ can equally be defined on $\mathcal{L} \odot \mathcal{R}$ for any complete lattices $\mathcal{L}=\left(L, \leq_{L}\right)$ and $\mathcal{R}=\left(R, \leq_{R}\right)$. The orderings present the decision maker with a rather complete picture of the situation; depending on the underlying goals and attitudes, he or she may exploit the information in various ways.

Consider, for instance, the set $\left([0,1]^{2}\right)^{2}$ together with, e.g., the normalized Euclidean distance function. For any value $(S(u, v), S(v, u))$ one can measure its distance to the external elements of each order. Such distances give graded information which is often more helpful for the decision maker than just the orderings 
themselves. For example, when $(S(u, v), S(v, u))=((0.1,0.77),(0.25,0.41))$, the distance $^{10}$ to FT is 0.44 and the distance to TF is 0.67 , which indicates a preference of $v$ over $u$. Likewise, the distances 0.62 and 0.52 to UK and KU respectively may indicate that the amount of available information is greater for " $v \geq u$ " than for " $u \geq v "$.

Note also that, as shown in Figure 3 (see the diagram on the bottom-left side), the distance to FT (respectively, to TF) of each one of KT, UT, FU, FK, is $1 / 2$ (respectively, $\sqrt{3} / 2$ ), while the distance to FT (to TF) of TK, TU, UF, KF, is $\sqrt{3} / 2$ (respectively, 1/2). This can be interpreted as follows: the elements on the middle layer do not give any evidence that $u \geq v$ or $u \leq v$, the elements on the second layer from below give more evidence that $u \leq v$, and the elements on the fourth layer provide more evidence that $u \geq v$. As Figure 3 shows, similar layered structures and distance values are also induced by the other orders (see the bottom-right side of this figure for $\leq_{k}$, the top-left side for $\leq_{t}^{\prime}$, and the top-right side for $\leq_{k}^{\prime}$ ).

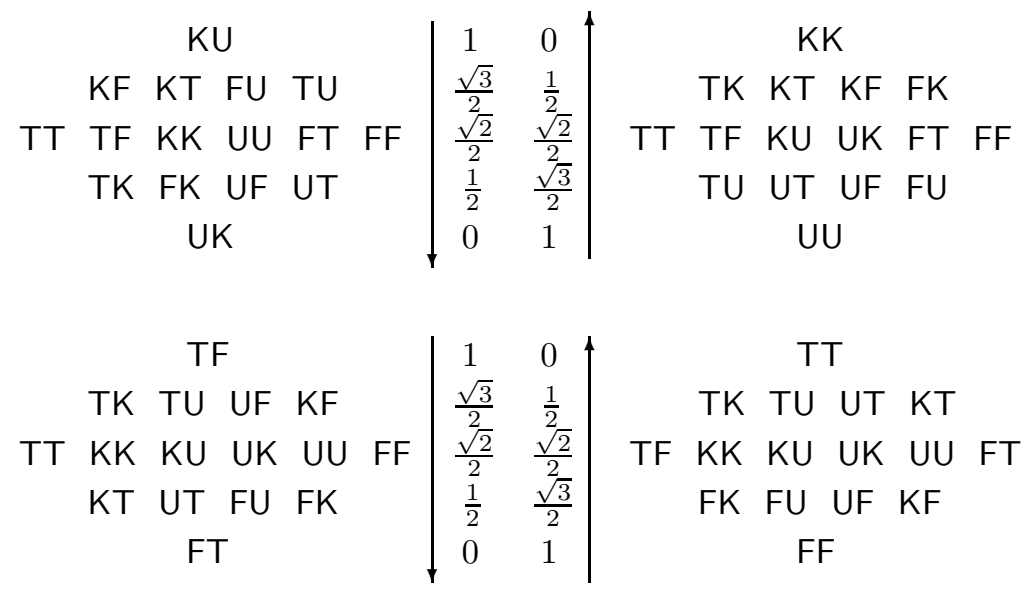

Fig. 3. Euclidean distances to the extreme elements of $\leq_{t}$ (bottom left), $\leq_{k}$ (bottom right), $\leq_{t}^{\prime}$ (top left), and $\leq_{k}^{\prime}$ (top right).

Figure 3 reveals a nice symmetry among the four diagrams: there are eight external elements each corresponding to a 'definite' state of affairs (TF and FT: strict preference; TT: indifference; FF: incomparability; KK, UU, UK, KU: information defect) and the eight remaining ones which float somewhat between the extremes (they are always in second or the fourth layer). Note also that the middle layer of each diagram always contains the six other external elements.

As the next proposition shows, the four order relations considered above preserve these distance considerations for every element of the underlying bilattice:

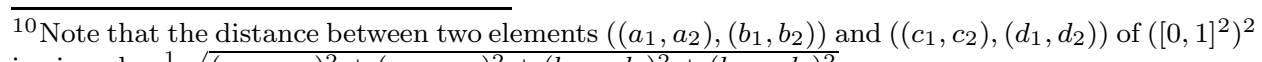
is given by $\frac{1}{2} \sqrt{\left(a_{1}-c_{1}\right)^{2}+\left(a_{2}-c_{2}\right)^{2}+\left(b_{1}-d_{1}\right)^{2}+\left(b_{2}-d_{2}\right)^{2}}$. 
Proposition 15. Let $\preceq$ be any one of the above four orders $\left(\leq_{t}, \leq_{k}, \leq_{t}^{\prime}, \leq_{k}^{\prime}\right)$ on $\left([0,1]^{2}\right)^{2}$, and let $d$ be the Euclidean distance function on it. Denote by 0 and 1 the $\preceq$-minimal element and the $\preceq$-maximal element, respectively. For every $u, v$ in $\left([0,1]^{2}\right)^{2}$, if $u \preceq v$ then $d(0, u) \leq d(0, v)$ and $d(u, 1) \geq d(v, 1)$.

Proof. We show the claim for $\leq_{t}$ and its minimum FT; the other cases are similar.

Let $u=\left(x_{1}, x_{2}\right)$ and $v=\left(y_{1}, y_{2}\right)$. If $u \leq_{t} v$ then $x_{1} \leq_{t} y_{1}$ and $x_{2} \geq_{t} y_{2}$, which means that $d_{E}\left(\mathrm{~F}, x_{1}\right) \leq d_{E}\left(\mathrm{~F}, y_{1}\right)$ and $d_{E}\left(\mathrm{~T}, x_{2}\right) \leq d_{E}\left(\mathrm{~T}, y_{2}\right)$, where $d_{E}$ denotes the Euclidean distance on $\mathbb{R}^{2}$. Thus, $d\left(\mathrm{FT},\left(x_{1}, x_{2}\right)\right)=\frac{1}{2} \sqrt{d_{E}\left(\mathrm{~F}, x_{1}\right)^{2}+d_{E}\left(\mathrm{~T}, x_{2}\right)^{2}} \leq$ $\frac{1}{2} \sqrt{d\left(\mathrm{~F}, y_{1}\right)^{2}+d\left(\mathrm{~T}, y_{2}\right)^{2}}=d\left(\mathrm{FT},\left(y_{1}, y_{2}\right)\right)$.

The above representation stands in sharp contrast to existing work relying on the conventions described in Note 3. Indeed, devising graded versions of the predicates $\mathbf{T}, \mathbf{F}, \mathbf{U}$ and $\mathbf{K}$ requires an explicit choice of how to model the conjunction in the right-hand sides of their defining equalities (8)-(11). In Refs. 60 and 61, two different choices involving different t-norms on the unit interval are put forward, each elaborately justified in its own terms. As our exposition reveals, however, this effort is altogether superfluous since it can be avoided by working with the original outranking information. As we have shown, rectangular bilattices offer a simple and natural way of encoding this information, even in cases that the argument in favour of a certain preference and the argument in disfavour of that preference are specified in terms of different ranges.

\section{Conclusion}

In this paper we introduced a general framework for uncertainty modeling, taking advantage of the new opportunities offered by bilattice-based structures. The 'traditional' approach of evaluating membership functions by values that are arranged in one (and usually total) order, is replaced here by more expressive 'two-dimensional' measurements that reflect different interpretations of the underlying orders, which may be applied simultaneously. The main benefits of this approach are, among others, the following:

(1) By not constraining ourselves to consistent elements only, a natural setting to represent and handle contradictions emerges (see Section 2).

(2) The constructs of rectangular bilattices relate IVFSs and IFSs within one uniform and general framework (see Section 3).

(3) The definition and representation of suitable logical connectives within our setting brings together results from both the theory of bilattices and $\mathcal{L}$-fuzzy set theory. Moreover, it raises many non-trivial questions regarding the interrelationships among the various alternatives (see Section 4).

(4) Some of the applicative aspects of our framework are demonstrated in the context of preference modeling, where we have introduced a generic declarative solution strategy to this problem. This solution fully exploits the order-theoretical 
ingredients of bilattice theory, and puts existing methods for preference modeling into a simple and unified perspective (see Section 5).

We conclude, therefore, that bilattice-based fuzzy sets provide a natural and attractive framework for the representation of uncertain and potentially conflicting information.

\section{Acknowledgements}

Chris Cornelis would like to thank the Research Foundation-Flanders for funding his research.

\section{References}

1. J. Goguen, L-fuzzy sets, Journal Math. Anal. Appl. 18 (1967) 145-174.

2. O. Arieli, C. Cornelis, G. Deschrijver and E. E. Kerre, Relating intuitionistic fuzzy sets and interval-valued fuzzy sets through bilattices, in Applied Computational Intelligence, eds. D. Ruan, P. D'Hondt, M. De Cock, M. Nachtegael and E. E. Kerre (World Scientific, 2004), pp. 57-64.

3. O. Arieli, C. Cornelis, G. Deschrijver and E. E. Kerre, Bilattice-based squares and triangles, in Proc. 8th European Conf. on Symbolic and Quantitative Approaches to Reasoning with Uncertainty (ECSQARU'05), ed. L. Godo (Springer, 2005), vol. 3571 of Lecture Notes in Artificial Intelligence, pp. 563-575.

4. C. Cornelis, O. Arieli, G. Deschrijver and E. E. Kerre, Uncertainty modeling by bilattice-based squares and triangles, IEEE Transactions on Fuzzy Sets and Systems In press.

5. M. L. Ginsberg, Multi-valued logics: A uniform approach to reasoning in artificial intelligence, Computer Intelligence 4 (1988) 256-316.

6. M. Fitting, Bilattices in logic programming, in Proc. 20th International Symposium on Multiple-Valued Logic, ed. G. Epstein (1990), pp. 238-246.

7. M. Fitting, Bilattices and the semantics of logic programming, Journal of Logic Programming 11 (1991) 91-116.

8. M. Fitting, Kleene's logic, generalized, Journal of Logic and Computation 1 (1992) 797-810.

9. M. Fitting, Kleene's three-valued logics and their children, Fundamenta Informaticae 20 (1994) 113-131.

10. F. Esteva, P. Garcia and L. Godo, Enriched interval bilattices and partial many-valued logics: an approach to deal with graded truth and imprecision, Uncertainty, Fuzziness and Knowledge-based Systems 2 (1994) 37-54.

11. G. Gargov, Knowledge, uncertainty and ignorance in logic: bilattices and beyond, Journal of Applied Non-Classical Logics 9 (1999) 195-283.

12. V. B. Tarassov, Lattice products, bilattices and some extensions of negations, triangular norms and triangular conorms, in Proc. Int. Conf. on Fuzzy Sets and Soft Computing in Economics and Finance (FSSCEF'04), eds. I. Batyrshin, J. Kacpzryk and L. Sherenetov (2004), vol. 1, pp. 272-282.

13. K. T. Atanassov, Intuitionistic fuzzy sets, (1983), vII ITKR's Session, Sofia (deposed in Central Sci.-Technical Library of Bulg. Acad. of Sci., 1697/84) (in Bulgarian).

14. R. Sambuc, Fonctions $\Phi$-floues. Application à l'aide au diagnostic en pathologie thyroidienne, (1975), ph. D. Thesis, University of Marseille.

15. M. Fitting, personal communication. 
16. C. Cornelis, K. T. Atanassov and E. E. Kerre, Intuitionistic fuzzy sets and intervalvalued fuzzy sets: a critical comparison, in Proc. 3rd Int. Conf. in Fuzzy Logic and Technology (EUSFLAT'03), eds. M. Wagenknecht and R. Hampel (2003), pp. 159163.

17. N. D. Belnap, How a computer should think, in Contemporary Aspects of Philosophy, ed. G. Ryle (Oriel Press, 1977), pp. 30-56.

18. N. D. Belnap, A useful four-valued logic, in Modern Uses of Multiple-Valued Logic, eds. G. Epstein and J. M. Dunn (Reidel Publishing Company, 1977), pp. 7-37.

19. O. Arieli and A. Avron, Reasoning with logical bilattices, Journal of Logic, Language, and Information 5 (1996) 25-63.

20. O. Arieli and A. Avron, Bilattices and paraconsistency, in Frontiers of Paraconsistent Logic, eds. D. Batens, C. Mortensen, G. Priest and J. V. Bendegem (Research Studies Press, 2000), pp. 11-27.

21. C. Cornelis, G. Deschrijver and E. E. Kerre, Square and triangle: reflections on two prominent mathematical structures for the representation of imprecision, Notes on Intuitionistic Fuzzy Sets 9 (2003) 11-21.

22. G. Deschrijver, C. Cornelis and E. E. Kerre, Square and triangle: a comparison, in Proc. Conf. on Information Processing and Management of Uncertainty in Knowledge-Based Systems (IPMU'04) (2004), pp. 1389-1396.

23. B. De Baets and R. Mesiar, Triangular norms on product lattices, Fuzzy Sets and Systems 104 (1999) 61-75.

24. A. Avron, The structure of interlaced bilattices, Journal of Mathematical Structures in Computer Science 6 (1996) 287-299.

25. J. M. Font and M. Moussavi, Note on a six-valued extension of three-valued logic, Journal of Applied Non-Classical Logic 3 (1993) 173-187.

26. M. Kondo, On the structure of weak interlaced bilattice, in Proc. 32th IEEE Int. Symp. on Multiple-Valued Logic (ISMVL'02) (2002), pp. 23-26.

27. L. A. Zadeh, Fuzzy sets, Information and Control 8 (1965) 338-353.

28. G. Takeuti and S. Titani, Intuitionistic fuzzy logic and intuitionistic fuzzy sets theory, Journal of Symbolic Logic 49 (1984) 851-866.

29. G. Deschrijver and E. E. Kerre, On the relationship between some extensions of fuzzy set theory, Fuzzy Sets and Systems 133 (2003) 227-235.

30. K. T. Atanassov and S. Stoeva, Intuitionistic L-fuzzy sets, Cybernetics and Systems Research 2 (1984) 539-540.

31. I. Grattan-Guinness, Fuzzy membership mapped onto interval and many-valued quantities, Zeitschrift für Mathematische Logik und Grundlagen der Mathematik 22 (1975) 149-160.

32. K. U. Jahn, Intervall-wertige Mengen, Math. Nach. 68 (1975) 115-132.

33. L. A. Zadeh, The concept of a linguistic variable and its application to approximate reasoning-I, Information Sciences 8 (1975) 199-249.

34. M. B. Gorzałczany., A method of inference in approximate reasoning based on intervalvalued fuzzy sets, Journal Math. Anal. Appl. 21 (1987) 1-17.

35. I. B. Türkşen, Interval valued fuzzy sets based on normal forms, Fuzzy Sets and Systems 20 (1986) 191-210.

36. C. Cornelis, G. Deschrijver and E. E. Kerre, Implication in intuitionistic and intervalvalued fuzzy set theory: Construction, classification, application, International Journal of Approximate Reasoning 35 (2004) 55-95.

37. D. Batens, C. Mortensen, G. Priest and J. V. Bendegem, eds., Frontiers of Paraconsistent Logic, Studies in Logic and Computation 8 (Research Studies Press, 2000).

38. W. A. Carnielli, M. E. Coniglio and I. Dóttaviano, eds., Paraconsistency: The Logical 
Way to the Inconsistent (Marcel Dekker, 2002), lecture Notes in Pure and Applied Mathematics 228.

39. J. Marcos, D. Batens and W. Carnielli, eds., Journal of Applied Logic, vol. 3 (Elsevier, 2005).

40. O. Arieli, Reasoning with different levels of uncertainty, Applied Non-Classical Logics 13 (2003) 317-343.

41. A. Pankowska and M. Wygralak, Intuitionistic fuzzy sets - an alternative look, in Proc. 3rd Int. Conf. in Fuzzy Logic and Technology (EUSFLAT'03), eds. M. Wagenknecht and R. Hampel (2003).

42. K. T. Atanassov, Remark on a property of the intuitionistic fuzzy interpretation triangle, Notes on Intuitionistic Fuzzy Sets 8 (2002) 8-37.

43. G. de Cooman and E. E. Kerre, Order norms on bounded partially ordered sets, Journal of Fuzzy Mathematics 2 (1994) 281-310.

44. G. Wagner, Vivid logic, Lecture Notes in Artificial Intelligence $\mathbf{7 6 4 .}$

45. P. Doherty, D. Driankov and A. Tsoukiàs, Partiality, para-consistency and preference modelling, (1992), iDA Research report Lith-IDA-R-92-18, Linköping University.

46. A. Tsoukiàs, A first-order, four valued, weakly paraconsistent logic and its relation to rough sets semantics, Foundations of Computing and Decision Sciences 27 (2002) $77-96$.

47. O. Arieli and A. Avron, The value of the four values, Artificial Intelligence 102 (1998) 97-141.

48. C. Cornelis, G. Deschrijver and E. E. Kerre, Classification of intuitionistic fuzzy implicators: an algebraic approach, in Proc. 6th Joint Conf. on Information Sciences (JCIS'01), eds. H. J. Caulfield, S. Chen, H. Chen, R. Duro, V. Honavar, E. E. Kerre, M. Lu, M. G. Romay, T. K. Shih, D. Ventura, P. P. Wang and Y. Yang (2002), pp. 105-108.

49. P. Hájek, Metamathematics of Fuzzy Logic (Kluwer Academic Publishers, Dordrecht, 1998).

50. E. Turunen, Mathematics behind fuzzy logic (Advances in Soft Computing, PhysicaVerlag, 1999).

51. C. C. Chang, Algebraic analysis of many valued logics, Trans. AMS93 (1958) 74-80.

52. S. Jenei, New family of triangular norms via contrapositive symmetrization of residuated implications, Fuzzy Sets and Systems 110 (2000) 157-174.

53. R. Brafman and U. Junker, eds., Multidisciplinary IJCAI-05 Workshop on Advances in Preference Handling (2005),

Online proceedings: http://wikix.ilog.fr/wiki/bin/view/Preference05/WebHome.

54. B. De Baets, M. Delgado, J. Fodor, F. Herrera, E. Herrera-Viedma and L. Martinez, eds., International Journal of Intelligent Systems, vol. 18(7) (2003), special issue on preference modeling and applications.

55. M. Roubens and P. Vincke, Preference modelling, Lecture Notes in Economics and Mathematical Systems $\mathbf{2 5 0 .}$

56. J. Fodor and M. Roubens, Fuzzy Preference Modelling and Multicriteria Decision Support (Kluwer, 1994).

57. B. Van de Walle, B. De Baets and E. E. Kerre, A plea for the use of Łukasiewicz triplets in the definition of fuzzy preference structures. (I). General argumentation, Fuzzy Sets and Systems 97 (1998) 349-359.

58. B. Van de Walle, B. De Baets and E. E. Kerre, A plea for the use of Lukasiewicz triplets in the definition of fuzzy preference structures. (II). The identity case, Fuzzy Sets and Systems 99 (1998) 303-310.

59. A. Tsoukiàs and P. Vincke, Extended preference structures in MCDA, in Multi-criteria 
Analysis, ed. J. Clímaco (Springer-Verlag, 1997), pp. 37-50.

60. P. Fortemps and R. Słowiński, A graded quadrivalent logic for ordinal preference modelling: Loyola-like approach, Fuzzy Optimization and Decision Making 1 (2002) 93-111.

61. P. Perny and A. Tsoukiàs, On the continuous extension of a four valued logic for preference modelling, in Proc. Conf. on Information Processing and Management of Uncertainty in Knowledge-Based Systems (IPMU'98) (1998), pp. 302-309.

62. A. Tsoukiàs, P. Perny and P. Vincke, From concordance/discordance to the modelling of positive and negative reasons in decision aiding, in Aiding Decisions with Multiple Criteria: Essays in Honor of Bernard Roy, eds. D. Bouyssou, E. Jacquet-Lagrèze, P. Perny, R. Slowiński and D. Vanderpooten (Kluwer, 2002), pp. 147-174.

63. A. Tsoukiàs and P. Vincke, A new axiomatic foundation of partial comparability, Theory and Decision 39 (1995) 79-114.

64. S. Jenei and B. De Baets, On the direct decomposability of t-norms on product lattices, Fuzzy Sets and Systems 139 (2003) 699-707.

65. U. Höhle, Commutative, residuated l-monoids, in Non-Classical Logics and Their Applications to Fuzzy Subsets, eds. U. Höhle and E. P. Klement (Kluwer Academic, Dordrecht, 1995), pp. 53-106.

\section{Proofs of the Propositions in Section 4}

Many proofs of the propositions in Section 4 are derived from similar proofs that appear in Ref. 4. In such cases, instead of repeating the corresponding proofs in their adjusted version, we just give the reference to the original text.

Notation 1. The mappings $\mathrm{pr}_{1}$ and $\mathrm{pr}_{2}$ on $L \times R$ are defined as follows: $\operatorname{pr}_{1}\left(x_{1}, x_{2}\right)=$ $x_{1}$ and $\operatorname{pr}_{2}\left(x_{1}, x_{2}\right)=x_{2}$.

Proof of Proposition 7. We shall show that if $\mathfrak{N}$ is an involutive negator on $\left(L \times R, \leq_{t}\right)$ then either $\mathfrak{N}\left(0_{\mathcal{L}}, 0_{\mathcal{R}}\right)=\left(1_{\mathcal{L}}, 1_{\mathcal{R}}\right)$ or $\mathfrak{N}\left(0_{\mathcal{L}}, 0_{\mathcal{R}}\right)=\left(0_{\mathcal{L}}, 0_{\mathcal{R}}\right)$ (Lemma 7 A); in the first case Formula (1) applies (Lemmas 7-B,C,D) and in the second case Formula (2) applies (Lemmas 7-E,F,G).

Lemma 7- $A$ : Let $\mathcal{L}=\left(L, \leq_{L}\right)$ and $\mathcal{R}=\left(R, \leq_{R}\right)$ be chains. For any involutive negator $\mathfrak{N}$ on $\left(L \times R, \leq_{t}\right)$ it holds that either $\mathfrak{N}\left(0_{\mathcal{L}}, 0_{\mathcal{R}}\right)=\left(0_{\mathcal{L}}, 0_{\mathcal{R}}\right)$ or $\mathfrak{N}\left(0_{\mathcal{L}}, 0_{\mathcal{R}}\right)=$ $\left(1_{\mathcal{L}}, 1_{\mathcal{R}}\right)$.

Proof: The proof is similar to the proof of Lemma 3-A in Ref. 4. Assume that $\mathfrak{N}\left(0_{\mathcal{L}}, 0_{\mathcal{R}}\right)=\left(x_{1}, 0_{\mathcal{R}}\right)$, where $x_{1}>_{L} 0_{\mathcal{L}}$. Then $\left(0_{\mathcal{L}}, 0_{\mathcal{R}}\right) \leq_{t}\left(x_{1}, 0_{\mathcal{R}}\right)$ and $\left(x_{1}, 1_{\mathcal{R}}\right) \leq_{t}\left(x_{1}, 0_{\mathcal{R}}\right)$, but $\left(0_{\mathcal{L}}, 0_{\mathcal{R}}\right)$ and $\left(x_{1}, 1_{\mathcal{R}}\right)$ are incomparable w.r.t. $\leq_{t}$. Since $\mathfrak{N}$ is decreasing and involutive, we obtain $\mathfrak{N}\left(0_{\mathcal{L}}, 0_{\mathcal{R}}\right) \geq_{t} \mathfrak{N}\left(x_{1}, 0_{\mathcal{R}}\right)=\left(0_{\mathcal{L}}, 0_{\mathcal{R}}\right)$ and $\mathfrak{N}\left(x_{1}, 1_{\mathcal{R}}\right) \geq_{t} \mathfrak{N}\left(x_{1}, 0_{\mathcal{R}}\right)=\left(0_{\mathcal{L}}, 0_{\mathcal{R}}\right)$. Hence the second component of both $\mathfrak{N}\left(0_{\mathcal{L}}, 0_{\mathcal{R}}\right)$ and $\mathfrak{N}\left(x_{1}, 1_{\mathcal{R}}\right)$ must be $0_{\mathcal{R}}$, thus $\mathfrak{N}\left(0_{\mathcal{L}}, 0_{\mathcal{R}}\right)$ and $\mathfrak{N}\left(x_{1}, 1_{\mathcal{R}}\right)$ are $\leq_{t^{-}}$ comparable. Now, since $\mathfrak{N}$ is decreasing and involutive, it also follows that $\left(0_{\mathcal{L}}, 0_{\mathcal{R}}\right)$ and $\left(x_{1}, 1_{\mathcal{R}}\right)$ are $\leq_{t}$-comparable, which is a contradiction. For the other cases of $\mathfrak{N}\left(0_{\mathcal{L}}, 0_{\mathcal{R}}\right) \in L \times R \backslash\left\{\left(0_{\mathcal{L}}, 0_{\mathcal{R}}\right),\left(1_{\mathcal{L}}, 1_{\mathcal{R}}\right)\right\}$, we obtain a contradiction in a similar way. Lemma 7-B: Let $\mathcal{L}=\left(L, \leq_{L}\right)$ and $\mathcal{R}=\left(R, \leq_{R}\right)$ be chains. For any involutive negator $\mathfrak{N}$ on $\left(L \times R, \leq_{t}\right)$ such that $\mathfrak{N}\left(0_{\mathcal{L}}, 0_{\mathcal{R}}\right)=\left(1_{\mathcal{L}}, 1_{\mathcal{R}}\right)$, the following holds for all 
$x_{1}$ in $L$ and $x_{2}$ in $R: \mathfrak{N}\left(x_{1}, 0_{\mathcal{R}}\right) \leq_{t}\left(1_{\mathcal{L}}, 1_{\mathcal{R}}\right), \mathfrak{N}\left(0_{\mathcal{L}}, x_{2}\right) \geq_{t}\left(1_{\mathcal{L}}, 1_{\mathcal{R}}\right), \mathfrak{N}\left(x_{1}, 1_{\mathcal{R}}\right) \geq_{t}$ $\left(0_{\mathcal{L}}, 0_{\mathcal{R}}\right)$ and $\mathfrak{N}\left(1_{\mathcal{L}}, x_{2}\right) \leq_{t}\left(0_{\mathcal{L}}, 0_{\mathcal{R}}\right)$.

Proof: Similar to that of Lemma 3-B in Ref. 4.

Lemma 7-C: Let $\mathcal{L}=\left(L, \leq_{L}\right)$ and $\mathcal{R}=\left(R, \leq_{R}\right)$ be chains. For any involutive negator $\mathfrak{N}$ on $\left(L \times R, \leq_{t}\right)$ such that $\mathfrak{N}\left(0_{\mathcal{L}}, 0_{\mathcal{R}}\right)=\left(1_{\mathcal{L}}, 1_{\mathcal{R}}\right)$, it holds that $\operatorname{pr}_{1} \mathfrak{N}\left(x_{1}, x_{2}\right)=$ $\operatorname{pr}_{1} \mathfrak{N}\left(x_{1}, 0_{\mathcal{R}}\right)$ and $\operatorname{pr}_{2} \mathfrak{N}\left(x_{1}, x_{2}\right)=\operatorname{pr}_{2} \mathfrak{N}\left(0_{\mathcal{L}}, x_{2}\right)$, for all $\left(x_{1}, x_{2}\right) \in L \times R$.

Proof: Similar to that of Lemma $3-\mathrm{C}$ in Ref. 4.

Lemma 7 - $D$ : Let $\mathcal{L}=\left(L, \leq_{L}\right)$ and $\mathcal{R}=\left(R, \leq_{R}\right)$ be chains and let $\mathfrak{N}$ be a negator on $\left(L \times R, \leq_{t}\right)$ such that $\mathfrak{N}\left(0_{\mathcal{L}}, 0_{\mathcal{R}}\right)=\left(1_{\mathcal{L}}, 1_{\mathcal{R}}\right)$. Furthermore, let the mappings $\mathcal{N}_{1}: L \rightarrow L$ and $\mathcal{N}_{2}: R \rightarrow R$ be defined by $\mathcal{N}_{1}\left(x_{1}\right)=\operatorname{pr}_{1} \mathfrak{N}\left(x_{1}, 0_{\mathcal{R}}\right)$ and $\mathcal{N}_{2}\left(x_{2}\right)=$ $\operatorname{pr}_{2} \mathfrak{N}\left(0_{\mathcal{L}}, x_{2}\right)$, for all $x_{1} \in L$ and $x_{2} \in R$. Then $\mathfrak{N}$ is involutive if and only if $\mathcal{N}_{1}$ and $\mathcal{N}_{2}$ are involutive negators on $\mathcal{L}$ and $\mathcal{R}$ respectively, and for all $\left(x_{1}, x_{2}\right)$ in $L \times R$ we have that $\mathfrak{N}\left(x_{1}, x_{2}\right)=\left(\mathcal{N}_{1}\left(x_{1}\right), \mathcal{N}_{2}\left(x_{2}\right)\right)$.

Proof: Similar to that of Lemma 3-D in Ref. 4.

Lemma 7-E: Let $\mathcal{L}=\left(L, \leq_{L}\right)$ and $\mathcal{R}=\left(R, \leq_{R}\right)$ be chains. For any involutive negator $\mathfrak{N}$ on $\left(L \times R, \leq_{t}\right)$ such that $\mathfrak{N}\left(0_{\mathcal{L}}, 0_{\mathcal{R}}\right)=\left(0_{\mathcal{L}}, 0_{\mathcal{R}}\right)$, the following holds for all $x_{1}$ in $L$ and $x_{2}$ in $R: \mathfrak{N}\left(x_{1}, 0_{\mathcal{R}}\right) \leq_{t}\left(0_{\mathcal{L}}, 0_{\mathcal{R}}\right), \mathfrak{N}\left(0_{\mathcal{L}}, x_{2}\right) \geq_{t}\left(0_{\mathcal{L}}, 0_{\mathcal{R}}\right), \mathfrak{N}\left(x_{1}, 1_{\mathcal{R}}\right) \geq_{t}$ $\left(1_{\mathcal{L}}, 1_{\mathcal{R}}\right)$ and $\mathfrak{N}\left(1_{\mathcal{L}}, x_{2}\right) \leq_{t}\left(1_{\mathcal{L}}, 1_{\mathcal{R}}\right)$.

Proof: Similar to that of Lemma 3-E in Ref. 4.

Lemma 7 -F: Let $\mathcal{L}=\left(L, \leq_{L}\right)$ and $\mathcal{R}=\left(R, \leq_{R}\right)$ be chains. For any involutive negator $\mathfrak{N}$ on $\left(L \times R, \leq_{t}\right)$ such that $\mathfrak{N}\left(0_{\mathcal{L}}, 0_{\mathcal{R}}\right)=\left(0_{\mathcal{L}}, 0_{\mathcal{R}}\right)$, it holds that $\operatorname{pr}_{1} \mathfrak{N}\left(x_{1}, x_{2}\right)=\operatorname{pr}_{1} \mathfrak{N}\left(0_{\mathcal{L}}, x_{2}\right)$ and $\operatorname{pr}_{2} \mathfrak{N}\left(x_{1}, x_{2}\right)=\operatorname{pr}_{2} \mathfrak{N}\left(x_{1}, 0_{\mathcal{R}}\right)$ for all $\left(x_{1}, x_{2}\right)$ in $L \times R$.

Proof: Similar to that of Lemma 3-F in Ref. 4.

Lemma 7 - $G$ : Let $\mathcal{L}=\left(L, \leq_{L}\right)$ and $\mathcal{R}=\left(R, \leq_{R}\right)$ be chains and let $\mathfrak{N}$ be a negator on $\left(L \times R, \leq_{t}\right)$ such that $\mathfrak{N}\left(0_{\mathcal{L}}, 0_{\mathcal{R}}\right)=\left(0_{\mathcal{L}}, 0_{\mathcal{R}}\right)$. Furthermore, define the mapping $\varphi: L \rightarrow R$ by $\varphi(x)=\operatorname{pr}_{2} \mathfrak{N}\left(x, 0_{\mathcal{R}}\right)$, for all $x \in L$. Then $\mathfrak{N}$ is involutive if and only if $\varphi$ is an isomorphism between $\mathcal{L}$ and $\mathcal{R}$, and for all $\left(x_{1}, x_{2}\right)$ in $L \times R$ we have that $\mathfrak{N}\left(x_{1}, x_{2}\right)=\left(\varphi^{-1}\left(x_{2}\right), \varphi\left(x_{1}\right)\right)$.

Proof: Assume first that $\mathfrak{N}$ is an involutive negator on $\left(L \times R, \leq_{t}\right)$ such that $\mathfrak{N}\left(0_{\mathcal{L}}, 0_{\mathcal{R}}\right)=\left(0_{\mathcal{L}}, 0_{\mathcal{R}}\right)$. Define the mappings $\varphi_{1}: R \rightarrow L$ and $\varphi_{2}: L \rightarrow R$ by $\varphi_{1}(x)=\operatorname{pr}_{1} \mathfrak{N}\left(0_{\mathcal{L}}, x\right)$ and $\varphi_{2}(x)=\operatorname{pr}_{2} \mathfrak{N}\left(x, 0_{\mathcal{R}}\right)$. We will show that $\varphi_{2}$ is an isomorphism between $\mathcal{L}$ and $\mathcal{R}$.

- Let $x$ and $x^{\prime}$ be distinct elements of $L$ and assume that $\varphi_{2}(x)=\varphi_{2}\left(x^{\prime}\right)=y$. Then, by definition of $\varphi_{2}$ and by Lemma 7 -E it follows that $\left(0_{\mathcal{L}}, y\right)=$ $\mathfrak{N}\left(x, 0_{\mathcal{R}}\right)=\mathfrak{N}\left(x^{\prime}, 0_{\mathcal{L}}\right)$. Thus, since $\mathfrak{N}$ is an involution, $\mathfrak{N}\left(0_{\mathcal{L}}, y\right)=\left(x, 0_{\mathcal{R}}\right)=$ $\left(x^{\prime}, 0_{\mathcal{L}}\right)$, which is a contradiction. Hence $\varphi_{2}$ is injective.

- Let arbitrarily $y \in R$. By Lemma 7-E it follows that there exists an $x \in L$ such that $\mathfrak{N}\left(0_{\mathcal{L}}, y\right)=\left(x, 0_{\mathcal{R}}\right)$. Since $\mathfrak{N}$ is involutive, $\mathfrak{N}\left(x, 0_{\mathcal{R}}\right)=\left(0_{\mathcal{L}}, y\right)$, so $\varphi_{2}(x)=y$. Hence $\varphi_{2}$ is surjective. 
- Finally we prove that for all $x, x^{\prime}$ in $L, x \leq_{L} x^{\prime} \Leftrightarrow \varphi_{2}(x) \leq_{R} \varphi_{2}\left(x^{\prime}\right)$. Assume first that $x \leq_{L} x^{\prime}$. Then $\left(x, 0_{\mathcal{R}}\right) \leq_{t}\left(x^{\prime}, 0_{\mathcal{R}}\right)$, so $\mathfrak{N}\left(x, 0_{\mathcal{R}}\right) \geq_{t} \mathfrak{N}\left(x^{\prime}, 0_{\mathcal{R}}\right)$. Hence $\varphi_{2}(x) \leq_{R} \varphi_{2}\left(x^{\prime}\right)$. Conversely, assume that $\varphi_{2}(x) \leq_{R} \varphi_{2}\left(x^{\prime}\right)$. By Lemma 7 -E it follows that there exist $y, y^{\prime}$ in $R$ such that $\left(0_{\mathcal{L}}, y\right)=\mathfrak{N}\left(x, 0_{\mathcal{R}}\right)$ and $\left(0_{\mathcal{L}}, y^{\prime}\right)=$ $\mathfrak{N}\left(x^{\prime}, 0_{\mathcal{R}}\right)$. So from $\varphi_{2}(x) \leq_{R} \varphi_{2}\left(x^{\prime}\right)$ it follows that $\left(0_{\mathcal{L}}, y\right) \geq_{t}\left(0_{\mathcal{L}}, y^{\prime}\right)$. Since $\mathfrak{N}$ is $\leq_{t^{-}}$-decreasing and involutive, we obtain that $\left(x, 0_{\mathcal{R}}\right) \leq_{t}\left(x^{\prime}, 0_{\mathcal{R}}\right)$, so $x \leq_{L} x^{\prime}$.

Now, by Lemma 7-F, for every element $\left(x_{1}, x_{2}\right)$ in $L \times R$ it holds that $\operatorname{pr}_{1} \mathfrak{N}\left(x_{1}, x_{2}\right)=$ $\operatorname{pr}_{1} \mathfrak{N}\left(0_{\mathcal{L}}, x_{2}\right)=\varphi_{1}\left(x_{2}\right)$ and $\operatorname{pr}_{2} \mathfrak{N}\left(x_{1}, x_{2}\right)=\operatorname{pr}_{2} \mathfrak{N}\left(x_{1}, 0_{\mathcal{R}}\right)=\varphi_{2}\left(x_{1}\right)$. In other words, for every $\left(x_{1}, x_{2}\right)$ in $L \times R, \mathfrak{N}\left(x_{1}, x_{2}\right)=\left(\varphi_{1}\left(x_{2}\right), \varphi_{2}\left(x_{1}\right)\right)$. Moreover, since $\mathfrak{N}$ is involutive, we have that $\mathfrak{N}\left(\mathfrak{N}\left(x_{1}, x_{2}\right)\right)=\left(\varphi_{1}\left(\varphi_{2}\left(x_{1}\right)\right), \varphi_{2}\left(\varphi_{1}\left(x_{2}\right)\right)\right)=\left(x_{1}, x_{2}\right)$, hence $\varphi_{1}=\varphi_{2}^{-1}$.

Assume conversely that $\varphi: L \rightarrow R$ is an isomorphism between $\mathcal{L}$ and $\mathcal{R}$ and define the mapping $\mathfrak{N}: L \times R \rightarrow L \times R$ by $\mathfrak{N}\left(x_{1}, x_{2}\right)=\left(\varphi^{-1}\left(x_{2}\right), \varphi\left(x_{1}\right)\right)$. Clearly, $\mathfrak{N}\left(0_{\mathcal{L}}, 0_{\mathcal{R}}\right)=\left(0_{\mathcal{L}}, 0_{\mathcal{R}}\right), \mathfrak{N}\left(0_{\mathcal{L}}, 1_{\mathcal{R}}\right)=\left(1_{\mathcal{L}}, 0_{\mathcal{R}}\right)$ and $\mathfrak{N}\left(1_{\mathcal{L}}, 0_{\mathcal{R}}\right)=\left(0_{\mathcal{L}}, 1_{\mathcal{R}}\right)$. Since $\varphi$ is increasing, $\mathfrak{N}$ is decreasing. Moreover, $\mathfrak{N}\left(\mathfrak{N}\left(x_{1}, x_{2}\right)\right)=\left(\varphi^{-1}\left(\varphi\left(x_{1}\right)\right), \varphi\left(\varphi^{-1}\left(x_{2}\right)\right)\right)=$ $\left(x_{1}, x_{2}\right)$, so $\mathfrak{N}$ is an involutive negator on $\left(L \times R, \leq_{t}\right)$.

Proof of Proposition 8. Similar to the proof of Proposition 8 in Ref. 4.

Proof of Proposition 9. Similar to the proof of Proposition 9 in Ref. 4.

Proof of Proposition 10. Assume first that $\mathfrak{N}\left(0_{\mathcal{L}}, 0_{\mathcal{R}}\right)=\left(1_{\mathcal{L}}, 1_{\mathcal{R}}\right)$. For $x=$ $\left(x_{1}, x_{2}\right), y=\left(y_{1}, y_{2}\right)$ in $L \times R$ we have,

$$
\begin{aligned}
\mathfrak{T}_{\mathfrak{N}}^{*}(x, y) & =\left(\mathcal{N}_{1}\left(\mathcal{T}\left(\mathcal{N}_{1}\left(x_{1}\right), \mathcal{N}_{1}\left(y_{1}\right)\right)\right), \mathcal{N}_{2}\left(\mathcal{S}\left(\mathcal{N}_{2}\left(x_{2}\right), \mathcal{N}_{2}\left(y_{2}\right)\right)\right)\right) \\
& =\left(\mathcal{T}_{\mathcal{N}_{1}}^{*}\left(x_{1}, y_{1}\right), \mathcal{S}_{\mathcal{N}_{2}}^{*}\left(x_{2}, y_{2}\right)\right) .
\end{aligned}
$$

Assume now that $\mathfrak{N}\left(0_{\mathcal{L}}, 0_{\mathcal{R}}\right)=\left(0_{\mathcal{L}}, 0_{\mathcal{R}}\right)$. For $x, y$ in $L \times R$ we have,

$$
\begin{aligned}
\mathfrak{T}_{\mathfrak{N}}^{*}(x, y) & =\left(\varphi^{-1}\left(\mathcal{S}\left(\varphi\left(x_{1}\right), \varphi\left(y_{1}\right)\right)\right), \varphi\left(\mathcal{T}\left(\varphi^{-1}\left(x_{2}\right), \varphi^{-1}\left(y_{2}\right)\right)\right)\right) \\
& =\left(\mathcal{S}_{\varphi}\left(x_{1}, y_{1}\right), \mathcal{T}_{\varphi^{-1}}\left(x_{2}, y_{2}\right)\right) .
\end{aligned}
$$

Proof of Proposition 11. Similar to the proof of Proposition 11 in Ref. 4. The proposition also follows from the fact (see Refs. 23, 64) that if the partial mappings of $\mathcal{T}$ are join-morphisms (which is always true if $\mathcal{T}$ is residuated ${ }^{65}$ ), then $\mathcal{T}$ is decomposable as the direct product of a t-norm on $\mathcal{L}$ and a t-norm on $\mathcal{R}$, i.e. $\mathcal{T}$ is $(\mathcal{L}, \mathcal{R})$-representable.

Proof of Proposition 12. Similar to the proof of Proposition 12 in Ref. 4.

Proof of Proposition 13. Similar to the proof of Proposition 15 in Ref. 4.

Proof of Proposition 14. Similar to the proof of Proposition 16 in Ref. 4. 